Review Article

\title{
The Gingiva from the Tissue Surrounding the Bone to the Tissue Regenerating the Bone: A Systematic Review of the Osteogenic Capacity of Gingival Mesenchymal Stem Cells in Preclinical Studies
}

\author{
Gamilah Al-Qadhi ${ }^{D},{ }^{1}$ Iman Aboushady $\mathbb{D}^{2},{ }^{2}$ and Niyaz Al-Sharabi $\mathbb{D}^{3}$ \\ ${ }^{1}$ Department of Basic Dental Sciences, Faculty of Dentistry, University of Science and Technology, Yemen \\ ${ }^{2}$ Department of Oral Biology, Faculty of Dentistry, Cairo University, Cairo, Egypt \\ ${ }^{3}$ Department of Clinical Dentistry, Faculty of Medicine, University of Bergen, Bergen, Norway \\ Correspondence should be addressed to Gamilah Al-Qadhi; gamilah.alqadhi@dentistry.cu.edu.eg
}

Received 16 October 2020; Revised 20 December 2020; Accepted 21 May 2021; Published 14 June 2021

Academic Editor: Stefania Cantore

Copyright (C) 2021 Gamilah Al-Qadhi et al. This is an open access article distributed under the Creative Commons Attribution License, which permits unrestricted use, distribution, and reproduction in any medium, provided the original work is properly cited.

\begin{abstract}
The current review aims to systematically assess the osteogenic capacity of gingiva-derived mesenchymal stem cells (GMSCs) in preclinical studies. A comprehensive electronic search of PubMed, Embase, Web of Science, and Scopus databases, as well as a manual search of relevant references, was performed in June 2020 without date or language restrictions. Eligibility criteria were the following: studies that compared mesenchymal stem cells (MSCs) derived from the gingiva with other MSC sources (in vitro or in vivo) or cell-free scaffold (in vivo) and studies that reported at least one of the following outcomes: osteogenic potential and new bone formation for in vitro and in vivo, respectively. Moreover, the assessment of included studies was conducted using appropriate guidelines. From 646 initial retrieved studies, 35 full-text articles were subjected to further screening and 26 studies were selected (20 in vitro studies and 6 in vivo studies). GMSCs showed great proliferation capacity and expressed recognized mesenchymal stem cell markers, particularly CD90. In vitro, MSC sources including GMSCs were capable of undergoing osteogenic differentiation with less ability in GMSCs, while most in vivo studies confirmed the capacity of GMSCs to regenerate bony defects. Concerning the assessment of methodological quality, in vitro studies met the relevant guideline except in five areas: the sample size calculation, randomization, allocation concealment, implementation, and blinding, and in vivo publications had probably low risk of bias in most domains except in three areas: allocation concealment, attrition, and blinding items.
\end{abstract}

\section{Introduction}

From autogeneic grafts to NanoBone materials, various therapeutic strategies to promote bone regeneration have been established over the past years. Autogeneic, allogeneic, and xenogeneic bone grafts, different forms of natural or synthetic bone graft substitutes, bioactive molecule-augmented bone graft substitutes, cell-based bone graft substitutes, and NanoBone materials have been reported in preclinical and clinical fields [1].
However, their implantation in bone regeneration has been associated with different complications. Although autogeneic grafting is considered the gold standard therapy, donor site pain, extrasurgery demand, increased surgical time, and postoperative infection were reported in several studies as drawbacks. Similarly, allogeneic grafting combined with the absence of osteogenic potential, risk of viral contamination, and lack of biological and mechanical properties due to the irradiation process required for antimicrobial purposes was reported. Further, the disadvantages of 
xenografts were the lack of osteogenic ability and risk of immune rejection [2].

In addition to the lack of osteoinductive capability, slow degradation rate, and lack of mechanical properties, high pressure and temperature processing have been demonstrated in the bone graft substitutes, such as calcium saltderived ceramics [3]. Augmentation of bone graft substitutes with growth factors such as bone morphogenetic protein results in accelerated bone healing [4]. It is clear that when materials are exposed to a biological environment, protein adsorption occurs immediately and subsequently mediates cell adhesion and proliferation processes. In comparison to conventional materials, nanomaterials may promote greater amounts of specific protein adsorption (e.g., albumin, laminin, collagen, fibronectin, and vitronectin). Not only the greater protein interactions but also the biomimetic size, larger surface area, and better mechanical properties make nanostructured material a good option to increase osteoblast functions (adhesion, proliferation, and differentiation) $[5,6]$. Yet, the application of dental nanomaterial may induce cellular toxicity and need more investigations [7].

Consequently, these limitations have led researchers to improve the existing techniques or to develop new ones. Among the proposed promising strategy for bone regeneration is stem cell-based therapy, significantly mesenchymal stem cells (MSCs). These cells are considered major key players in transitional regenerative medicine [8], mainly due to their differentiation capacity into specific cell types, profuse secretion of soluble growth factors and cytokines, migration and homing potential to the site of injury, immunomodulatory effects on innate and adaptive immune cells, and anti-inflammatory effects [9].

Immunomodulatory and anti-inflammatory effects occur through cell-cell interactions or paracrine activities. Interferon-gamma (IFN $\gamma$ ) combined with Tumor Necrosis Factor (TNF) or Interleukin-1 (IL-1) stimulates MSCs to produce chemokine receptor ligands such as CXC-Chemokine Receptor 3 (CXCR3) ligand, which in turn recruit $\mathrm{T}$ cells resulting in the suppression of proliferation and activity of $\mathrm{T}$ cells in their local environment. Besides, MSCs mediate immunosuppression by secretion of secretome including cytokines, growth factors, anti-inflammatory factors, and exosomes that inhibit the proliferation and function of proinflammatory cells, such as T Helper 1 (TH1) and/or TH17 cells, macrophages, neutrophils, Natural Killer (NK) cells, and B cells, and stimulate anti-inflammatory cells, such as macrophages and regulatory $\mathrm{T}$ and $\mathrm{B}$ cells. Further, antiinflammatory cells inhibit the function of proinflammatory cells and subsequently promote tissue healing [10].

MSCs can be used alone or in combination with a scaffold or carrier, which act as an extracellular template allowing stem cells to attach, proliferate, migrate, and differentiate into target cells. Various options in scaffold designing have been developed to direct and enhance osteogenic differentiation, for example, architecture modification (pore size, stiffness, and topography) [6], chemical stimulation through altering and adjusting the ratio of Hydroxyapatite (HA) to tricalcium phosphate (TCP) [11], biochemical stimulation by introduction of bioactive factors like dexamethasone or vitamin $\mathrm{C}$
[12], bone-specific growth factors [13], or addition of mineral fillers like Dicalcium Phosphate Dihydrate (DCPD) and hydraulic Calcium Silicate (CaSi) into Polylactic Acid (PLA) scaffolds [14]. More recently, a growing interest in $3 \mathrm{D}$ bioprinting techniques led to opening a new chapter for the production of the bioactive scaffold in bone regeneration [15].

Furthermore, mechanical stimulation can be used to enhance the proliferation and differentiation of MSCs into specific cell types [16]. In particular, the mechanical strain and biological fluid flow are converted into biochemical signals and then integrated into cellular responses through mechanotransduction. In bone tissue, osteocytes act as sensory cells responsible for this mechanotransduction, while osteoblasts and osteoclasts are the effector cells [17].

Several studies have been conducted using bone marrow mesenchymal stem cells (BMSCs) as a gold standard option for bone defects [18-20] and other dental MSC sources $[21,22]$. Nevertheless, harvesting inadequate numbers of BMSCs, long expansion culture time, pain, and morbidity that may occur following the aspiration and collection processes still represent a major issue [23]. Therefore, alternative sources from other locations with a sufficient number of cells and easy isolation procedures have prospected. MSCs from oral tissues, including the dental pulp of permanent and deciduous teeth, periodontal ligaments, dental follicles, and dental papillae, were highly committed to differentiate into osteoblasts and bone cell precursors [24]. Furthermore, MSCs from diseased oral tissue, such as the human periapical cysts [14], inflamed dental pulp, and gingiva [25], provide a new tool for bone regeneration.

One of the attractive dental MSC sources is the gingiva. It is a part of the oral mucosal tissue, which surrounds and supports the teeth and alveolar bone and has essential roles ranging from acting as a mucosal barrier to participating in oral mucosal immunity. The gingiva also represents the biomedical waste that occurs because of common dental procedures such as tooth removal, gum or periodontal surgery, and dental implant surgery [26]. Recently, stem cells isolated from the gingiva captivate many researchers in the regenerative medicine field to investigate their features and ability to regenerate tissue, including bone [27].

A novel population of stem cells from gingival tissue called gingiva-derived mesenchymal stem cells (GMSCs) was isolated from humans and successfully verified by many researchers $[28,29]$. Certainly, gingivae contain both neural crest-derived MSCs (90\%) and mesoderm-derived MSCs (10\%) with distinct stem cell properties. From clinical and laboratory aspects, the gingiva is considered an easily accessible convenient source with a less-invasive biopsy-taking technique and it is attainable to isolate sufficient numbers of MSCs from gingival tissue depending on their high proliferation rate [30].

In addition to their trilineage differentiation ability, selfrenewal, and expression of mesenchymal surface markers, they exhibited immunomodulatory and anti-inflammatory properties that make them an attractive source for regenerative application [28]. These cells met the standard minimal criteria proposed by the International Society for Cellular 
Therapy [31]. Along with bone regeneration, previous studies have indicated that GMSCs had the capacity to regenerate the nerve [32,33], muscle [34], tendon [35], skin [36], and cartilage and synovial tissues [37].

Equally important, the systematic reviews are ordinary procedures in clinical trials to assess the clinical efficiency of certain interventions while systematic reviews in the preclinical research community are still uncommon and need more attention to improve preclinical studies and in turn determine the first step in translating research from bench to patient [38].

Considering the above background, the current study is aimed at systematically reviewing the efficacy of applying GMSCs, as a newly introduced source, in bone regeneration in preclinical studies. This systematic review based on the following PICOS question: problem/population: bone defect in animal models (in vivo) and cell culture models (in vitro); intervention: GMSCs for both in vitro and in vivo; comparator or control: comparator: other sources (in vitro)/comparator or control: other sources and cell-free groups (in vivo); outcome: osteogenic capacity; and study design: preclinical studies. All items of PICOS have been used to formulate the following research question: will gingivaderived mesenchymal stem cells be considered comparable or alternative to other MSC sources regarding bone regeneration in preclinical studies?

\section{Materials and Methods}

The current systematic review was conducted in accordance with the following guideline: Preferred Reporting Items for Systematic Reviews and Meta-Analyses (PRISMA) [39] (see supplementary material I for the PRISMA checklist).

2.1. Protocol Registration. The protocol of the current study was registered at the CAMARADES (Collaborative Approach to Meta-Analysis and Review of Animal Data from Experimental Studies) website at the following link: https://drive.google .com/file/d/1fkC_zkxGueWPyguYHAUH2SPLnJkwI3gK/view.

2.2. PICOS Question. The current systematic review was designed to answer the following question: will gingivaderived mesenchymal stem cells be considered comparable or alternative to other MSC sources regarding bone regeneration in preclinical studies?

2.3. Eligibility Criteria. The eligibility criteria were established depending on the PICOS items. Therefore, any study that met the inclusion criteria was added as an eligible potential study. To be included, in vivo studies should consider the bone as a targeting area, and bone defects were induced in animals either by surgery or by disease regardless of species, age, and sex, as well as in vitro culture studies that compared MSCs derived from the gingiva and other MSC sources, in vivo studies that compared MSCs derived from the healthy gingiva and cell-free scaffold or other MSC sources, and studies that reported at least one of the following outcomes: new bone formation (in vivo) and osteogenic potential (in vitro).

On the other hand, publications that have the following criteria were excluded: targeted areas other than the bone, including the skin and gingiva, irrelevant intervention such as the inflamed gingiva, epithelial gingival cells or gingival fibroblasts, or induced pluripotent stem cells, as well as studies using derivatives of GMSCs such as Extracellular Vesicles (EVs), secretome, gene modification and cocultured cells, single-arm studies, reviews, opinion, and case studies.

2.4. Search Sources and Strategy. The popular electronic databases were systematically searched to identify the potential preclinical papers that assess the efficiency of GMSCs in regenerating the bone defects or osteodifferentiation in vivo and in vitro, respectively. The research was carried out in June 2020 and included the following databases: PubMed/MEDLINE, Embase, Web of Science, and Scopus, as well as a manual search of the reference lists of the relevant studies.

Based on "A step-by-step guide to systematically identify all relevant animal studies" [40], the research strategy was designed according to the type of the research engine using the following Search Component (SC) terms (medical subject headings (MeSH) terms, EMTREE, and free keywords) without date and language restrictions: SC1 (problem): (bone defect OR bone degradation OR bone disease OR bone disorder OR bone loss OR bone deformation OR bone destruction OR bone injury OR bone fracture) AND SC2 (intervention): (gingival mesenchymal stem cell OR gingival mesenchymal stem cells OR gingiva derived mesenchymal stem cell OR gingival tissue derived mesenchymal stem cells OR gingiva derived stromal cell OR gingiva derived stromal cells OR multipotent gingival stromal cell OR multipotent gingiva stromal cells OR multipotent gingiva progenitor cells OR gingiva stem cells) AND SC3 (control or compotator): (unloaded scaffold OR cell free scaffold) AND SC4 (outcome): (bone regeneration OR bone repair OR new bone formation OR bone healing OR bone tissue engineering OR bone remodeling OR osteogenesis OR osseointegration OR osteoconduction, osteogenic capacity (capability) OR osteogenic differentiation OR osteogenic potential) AND SC5 (study design): (animal model OR animal models OR experimental animal OR experimental animals OR laboratory animal OR laboratory animals OR in vivo study $\mathrm{OR}$ in vivo study $\mathrm{OR}$ in vitro study $\mathrm{OR}$ in vitro $\mathrm{OR}$ in vitro studies $\mathrm{OR}$ in vitro technique $\mathrm{OR}$ in vitro techniques OR cell culture technique OR cell culture method OR cell culture techniques OR culture technique OR culture techniques OR preclinical study) (see supplementary material II for the detailed search strategy).

2.5. Study Selection. All retrieved citations were imported and combined into the Mendeley folder, and the duplicated results were removed. Following that, two independent researchers (Al-Qadhi and Aboushady) did two phases of selection with specific exclusion items per phase. The first phase involved skimming the title and abstract of retrieved articles and then excluded the irrelevant studies, and the second phase involved scanning the full texts and excluded the irrelevant studies.

\subsubsection{Exclusion Criteria of Selection Phase I}

(i) Review, case report, and expert opinion

(ii) Irrelevant intervention, outcome, and problem 
(iii) Presence of any cofactors

(iv) Single-arm studies

\subsubsection{Exclusion Criteria of Selection Phase II}

(i) Review, case report, and expert opinion

(ii) Irrelevant intervention, outcome, and problem

(iii) Presence of any cofactors

(iv) Absence of MSC source comparator

(v) Mixing cells into one culture

(vi) Derivatives of a source of interest

In case of discrepancies, the two reviewers discussed the item and Al-Sharabi made the final decision. The reasons for exclusion were discussed and documented.

2.6. Data Extraction and Data Items. Data was collected and extracted using a preformed table designed by the authors. For in vitro culture studies, the following items were taken into consideration: study ID (author, year of publication), study design characteristics (experimental groups, source of stem cells, type of isolation method, type of culture medium, osteogenic induction period, density of cells/well, and type of scaffold or carrier if present), and outcome measures (proliferation potential, characterization of MSCs, and osteogenic differentiation).

Similarly, for in vivo studies, the following items were reported: study ID (author, year of publication), study design characteristics (experimental groups), animal model characteristics (species, gender, age, weight, and total numbers), intervention characteristics (source of stem cells, dose, mode of delivery, type of scaffold or carrier, and fate tracing), defect characteristics (site, size, way of induction, and time between induction and treatment), and outcome measures (characterization of MSCs, observation time points, method of analysis, and new bone formation).

2.7. Critical Appraisal of Individual Studies. To date, there is no specific risk-of-bias tool for quality assessment of in vitro culture experiments; therefore, we used the guidelines for reporting preclinical in vitro culture studies on dental materials based on the modification of the CONSORT checklist [41]. For in vivo studies, the risk of bias in included studies was evaluated using the Systematic Review Center for Laboratory Animal Experimentation (SYRCLE) risk-of-bias tool for animal intervention studies based on the Cochrane Collaboration RoB Tool with modification of some points [42]. The Health Assessment Workplace Collaborative (HAWC) online tool was used to manage the research process [43].

2.8. Summary Measures. To measure the primary outcome in in vitro culture studies, evidence of the formation of mineralized nodules was confirmed by Alizarin red staining or by the expression of osteogenic markers. Along the same line, outcome measures for in vivo studies were represented by the new bone formation that was assessed at least by histology, histochemistry, radiography, or gene expression of osteogenic markers.

2.9. Synthesis of Results. In the current systematic review, both outcome measures were performed in a qualitative description manner. The qualitative data synthesis was implanted because there was a marked variation in the way of presenting data among studies. Although most studies used the same outcome investigation method, some of them did not provide quantitative data; some of them used specific markers that differ from others and so on. Thus, the authors decided to illustrate the results in a qualitative manner rather than through a meta-analysis by trying the best to categorize data in a relevant chart.

\section{Results}

3.1. Study Selection. The followed search strategy resulted in the identification of a total of 646 possible relevant studies. Particularly, PubMed, Embase, Web of Science, and Scopus database searching provided 638 citations and manual searching identified additional 8 citations. Then, duplicated records were removed, resulting in 419 articles, and were subjected to skimming based on titles and abstracts. During this selection phase, 384 studies were excluded because they did not meet the criteria. Following that, 35 full-text remaining articles were selected for eligibility, and of these, 9 studies were excluded. The reason for exclusion was found in the supplementary material (see supplementary material III for the excluded studies with reasons). The previous steps are represented by the PRISMA flowchart (Figure 1).

\subsection{Characteristics of In Vitro Included Studies}

3.2.1. Methods. A total of 26 studies were selected for the current systematic review. Of these, 20 in vitro were culture studies [44-63] and 6 were in vivo studies [64-69]. All papers were published in the English language, and the date of publication started in 2009. Regarding in vitro culture studies, most papers used humans as a source of MSCs except four studies that used animal sources such as mice $[44,45]$, pigs [46], and horses [47]. Concerning in vivo studies, all studies used humans as a source of MSCs except one study that used rabbits [48].

An enzymatic method of GMSC isolation was used in all in vivo and in vitro culture studies except in 2 in vitro culture studies where the outgrowth isolation method was used $[49,50]$. In three in vitro studies, homogenous GMSCs were obtained after the isolation methods by colonyforming units [51] and fluorescence-activated cell sorting (FACS) [49], while a single-cell cloning method was used in only one in vivo study [52]. The osteogenic induction medium was used in all in vitro culture studies with an induction period that ranged from 14 to 28 days. The density of cells ranged from $2 \times 10^{3}$ to $2 \times 10^{6}$. Seven studies used scaffolds or carriers, commonly tricalcium phosphate (TCP) $[49,53-55]$ and alginate [56-58]. Six studies of 20 studies confirmed the culture results by an ectopic bone formation (Table 1). 

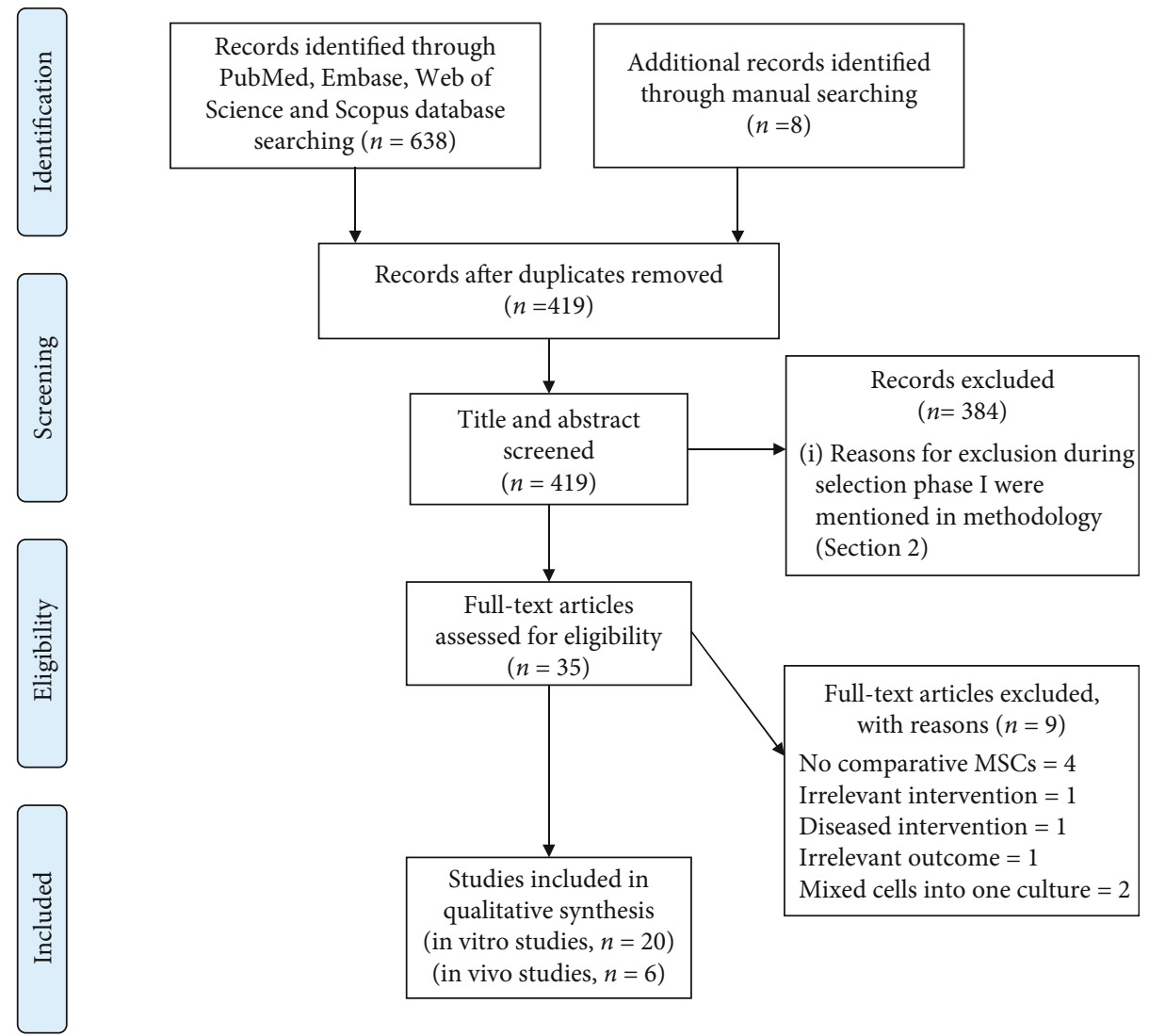

FIGURE 1: Flow diagram showing the different phases of literature screening for the systematic review process (editable file: PRISMA flow diagram, Liberati et al. 2009).

3.2.2. Type of Interventions. All studies compared GMSCs with dental or nondental MSC sources. In detail, studies compared GMSCs with periodontal ligament stem cells (PDLSCs) [59]; with dental pulp stem cells (DPSCs) $[50,54,60]$; with BMSCs [44, 49, 53, 55]; with buccal fat pad-derived mesenchymal stem cells (BFPMSCs) [53]; with PDLSCs and BMSCs [56, 57]; with PDLSCs and DPSCs [61]; with PDLSCs and dermal stem cells (DSCs) [55]; with PDLSCs and subcutaneous mesenchymal stem cells (ScMSCs) [47]; with BMSCs and submandibular salivary gland-derived mesenchymal stem cells (SSMSCs) [62]; with PDLSCs, DPSCs, and BMSCs [49]; with PDLSCs, BMSCs, adipose-derived stem cells (ADSCs), and periosteumderived mesenchymal stem cells (PSCs) [46]; and with PDLSCs, DPSCs, DFSCs, BMSCs, ADSCs, and umbilical cord mesenchymal stem cells (UCMSCs) [57] (Table 2).

3.2.3. Identification of Interventions. The proliferation rate of GMSCs was significantly higher in 10 studies compared to other MSC sources $[44,50,51,53,55-57,59,62,63]$. However, they were proliferated effectively in the remaining studies. Note that 3 studies did not perform the proliferation assessment $[58,64,65]$. MSCs must be characterized by their morphological appearance and functionally identified by differentiating into adipocytes, chondroblasts, and osteoblasts as well as phenotypically by expressing MSC surface markers CD29, CD73, CD90, and CD105, with lacking expression of CD45, CD34, CD14 or CD11b, CD79 alpha or CD19, and human leukocyte antigen-antigen D-related (HLA-DR) surface molecules [31].

Clearly, 10 studies verified GMSCs by using three ways of ISCT identification and approved MSC characteristics $[44,46,47,49,50,53-55,62,63]$. It is worth mentioning that some studies implemented three ways of ISCT, but two out of three differentiation assays were applied; for instance, GMSCs differentiated into osteoblasts and adipocytes in the following studies $[59,61,65]$ and into osteoblasts but not adipocytes in one study [46]. Besides, some articles utilized one out of three required differentiation analyses and revealed that GMSCs differentiated into osteoblasts only $[57,58,64]$. Two studies did not perform multilineage functional verification $[45,60]$.

Most studies carried out immunophenotype analysis and confirmed that all MSC sources were positively expressed MSC markers with the percentage of $>95 \%$ and were negative for hematopoietic markers with the percentage of $<5 \%$, excluding the two studies that did not provide sufficient CD marker analysis [57, 58], and one study did not establish the analysis [62] (Table 3).

3.2.4. Primary Outcomes. To measure the primary outcome of in vitro culture studies, osteogenic differentiation was confirmed by the formation of mineralized nodules using a histological staining method, particularly Alizarin red staining, and/or by the expression of osteogenic markers. All in vitro culture studies confirmed that MSC sources 


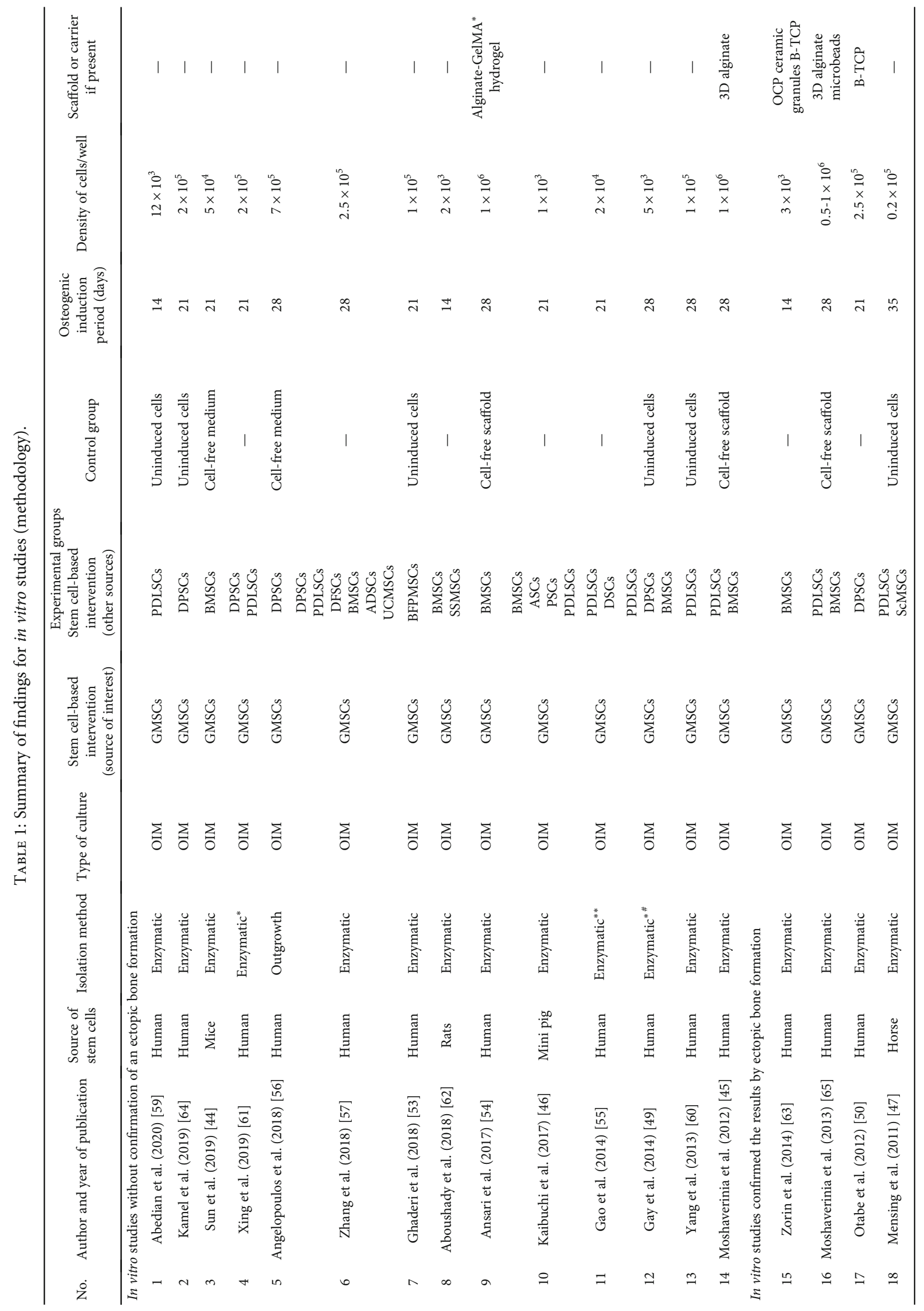




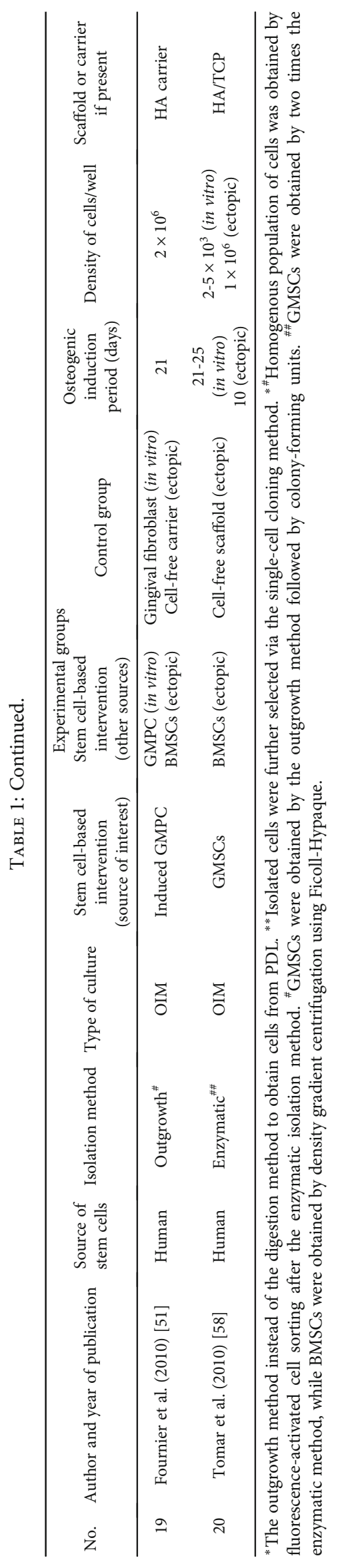




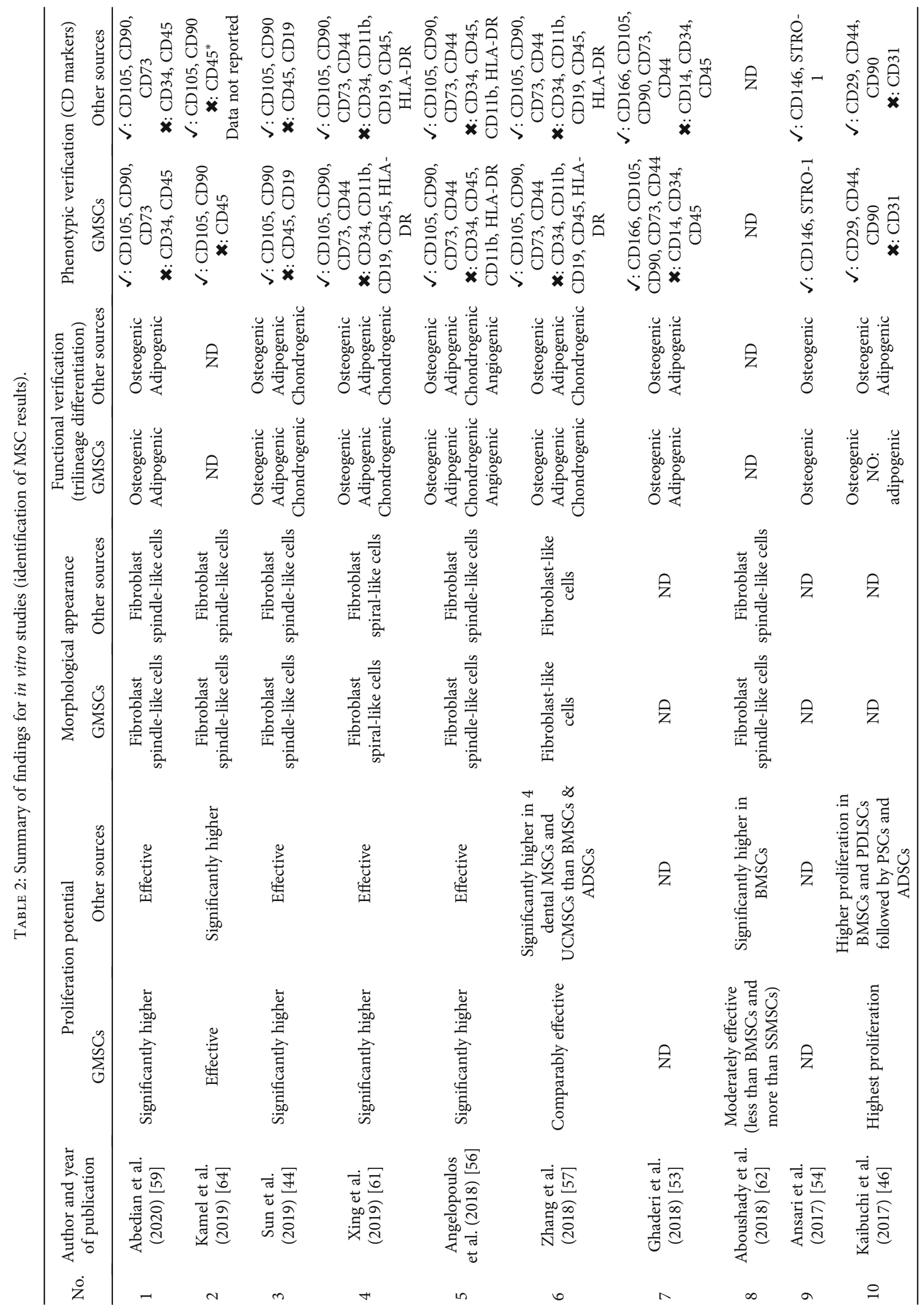




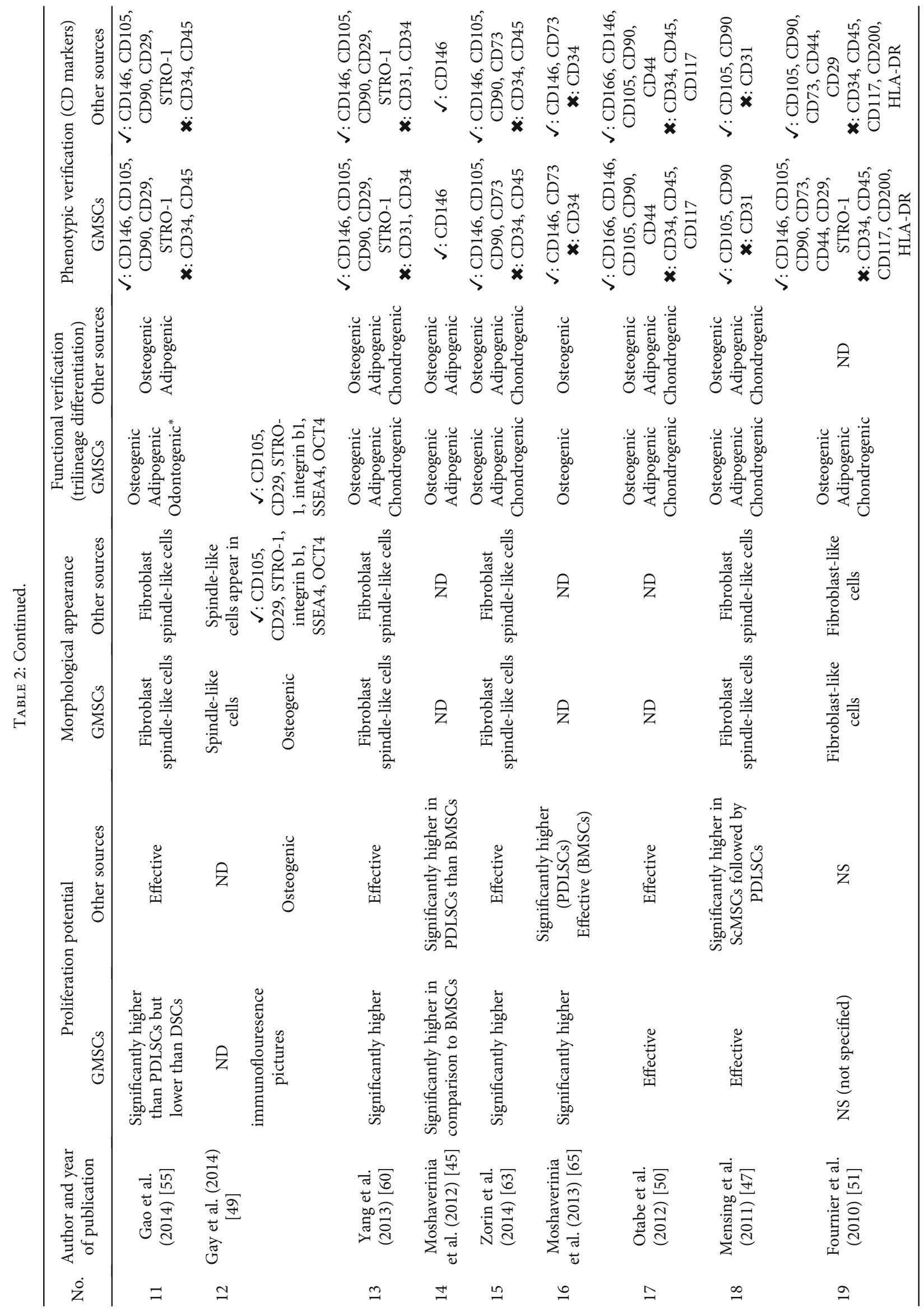




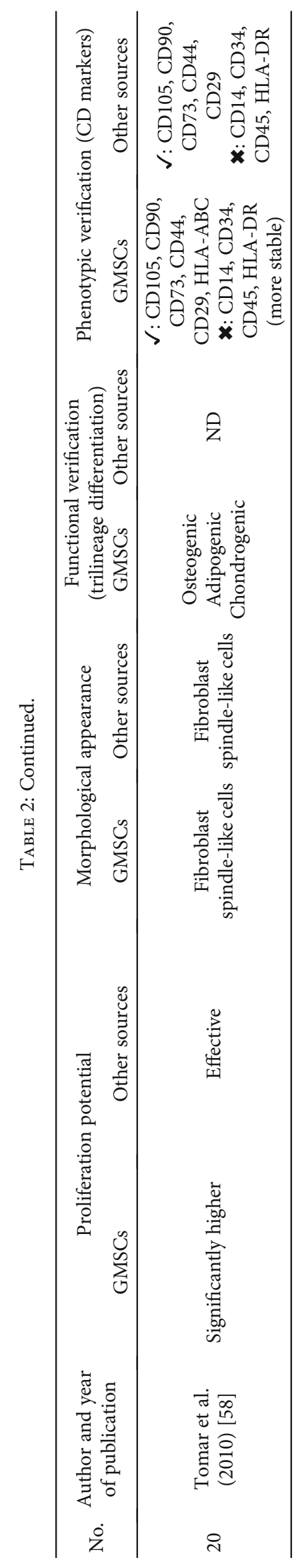




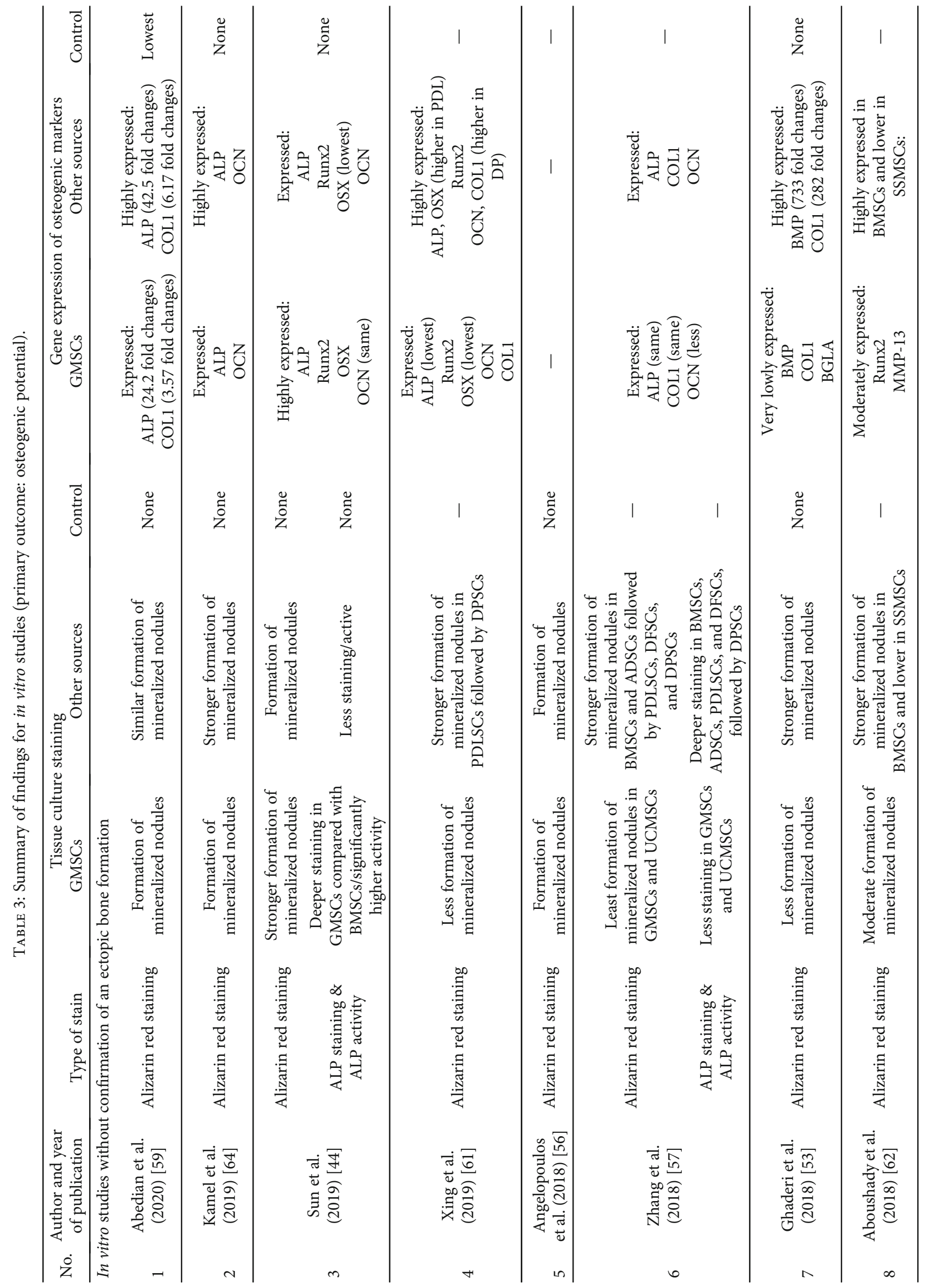




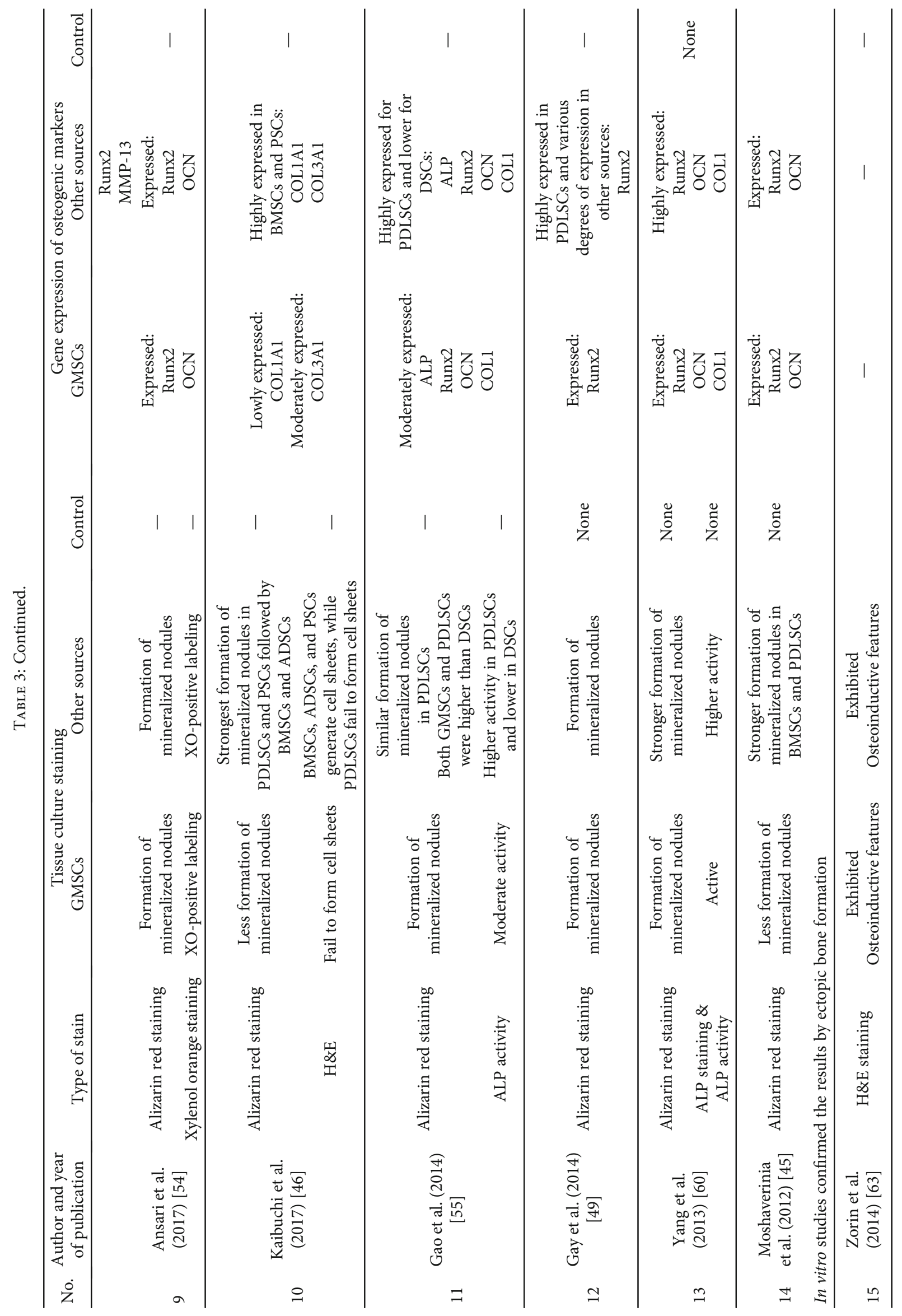



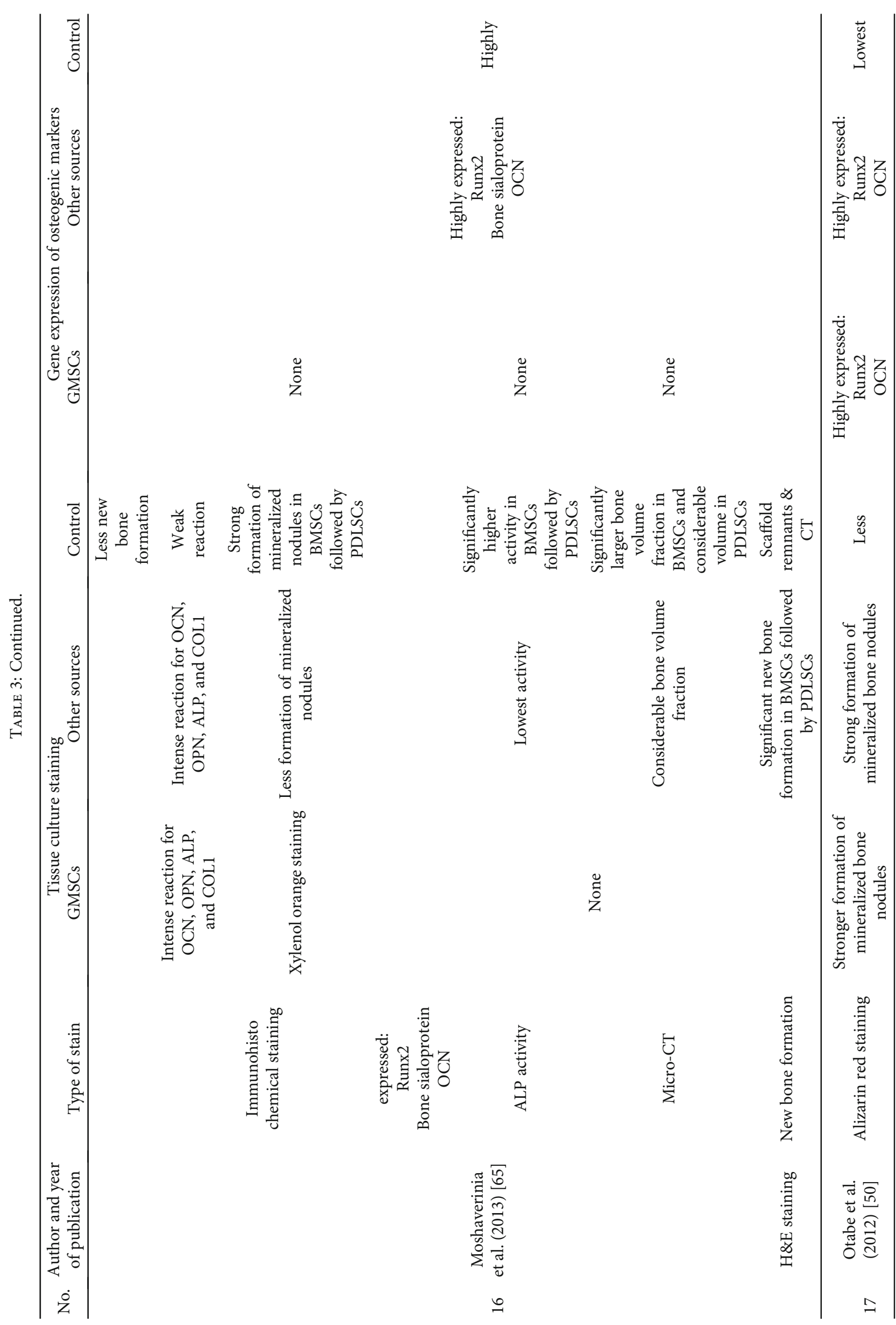


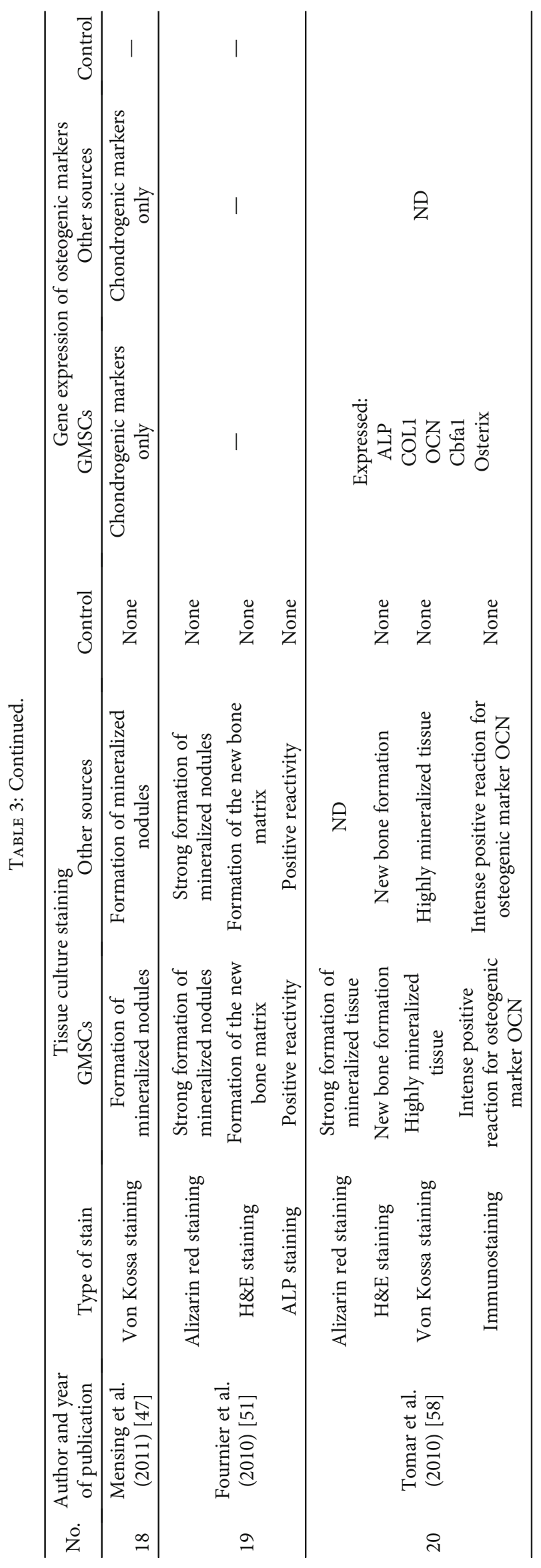


including GMSCs were capable of undergoing osteogenic differentiation with different degrees. Obviously, eight articles reported that GMSCs had a lower degree of osteogenic potential than other MSC sources [47, 51, 56, 57, 60, 62, 63, 65].

However, similar numbers of articles stated that both GMSCs and other MSC sources formed calcified nodules without determining which one is stronger than the other $[46,49,50,53,55,58,59,64]$. Only two studies have approved that GMSCs had a stronger ability to form mineralized nodules [44, 54]. One study reported that GMSCs had a moderate osteogenic capability particularly, lower than BMSCs and higher than SSMSCs [62], and another study showed that GMSCs had a similar osteogenic ability to PDLSCs, higher than DSCs [55].

Moreover, the majority of studies conducted additional confirmation methods by using stains such as alkaline phosphatase (ALP), Von Kossa, H\&E, and immunochemical stains or by gene expression of osteogenic markers. All articles that used gene expression analysis clarified that MSC sources, including GMSCs, expressed the following markers: ALP, Runt-Related Transcription Factor 2 (Runx2), Osteonectin (OCN), COL1, and Osterix (OSX), and the results were in agreement with staining results (Table 3 ).

\subsection{Characteristics of In Vivo Studies}

3.3.1. Methods. Due to limitations in the number of animal studies comparing GMSCs with other sources, the authors decided to include animal studies that compare GMSCs with the control groups as well. In addition to negative controls (scaffolds without stem cells) that were used in all in vivo studies, GMSCs were compared with BMSCs [48] and with PDLSCs and BMSCs [66]. Different animal species were used to create the bony defect, rabbits [48], mice [66-68], dogs [69], and rats [52]. The dose ranged from $1 \times 10^{6}$ to $4 \times 10^{6}$. Local application of MSCs was documented in four studies $[48,52,66,69]$, while two studies $[67,68]$ used a systemic delivery. Green fluorescent protein (GFP) was used as a fate tracer in four studies [52, 67-69], whereas PKH26 was used in one study [48], and no fate tracer was applied in one study [66]. Different locations for surgically created defects, including the tibia, maxilla, mandible, and calvaria with defect sizes that ranged from $0.5 \mathrm{~mm}$ to $6.0 \mathrm{~mm}$, were used. Besides, various scaffolds include NanoBone [48], Modified Eagle's Medium ( $\alpha$-MEM) [67, 68], alginate [66], cell sheet [69], and type 1 collagen (COL1) gel [52]. There was no study that mentioned the time between induction and application of intervention, except one [69] (Table 4).

3.3.2. Types of Interventions. All studies compared GMSCs with the cell-free scaffold. From 6 studies, one study compared GMSCs to BMSCs [48], and another study compared between GMSCs, BMSCs, and PDLSCs [66].

3.3.3. Identification of Interventions. Four animal studies verified MSCs using three ways of ISCT characterization items. However, one paper affirmed one of three differentiation lineages [66], and one paper did not establish the multilineage differentiation analysis [48] (Table 5).
3.3.4. Results of the Primary Outcome. The area of newly formed bone was detected in animal studies using Hematoxylin and Eosin (H\&E) staining and/or Masson Trichrome (MT) or immunohistochemical staining. In terms of in vivo studies, more newly formed bone areas in GMSC groups were detected by H\&E staining [48, 52, 67-69] with the exception of one article indicating that GMSCs had lower new bone formation in comparison to PDLSCs and BMSCs [66]. These results were validated by an abundance of red color indicating mature bone formation $[48,67,68]$ and strong expression of GFP, Osteopontin (OPN), and COL1 [52] (Table 5).

3.4. Critical Appraisal within Sources of Evidence. All in vitro culture studies lacked particularly five items: the sample size calculation, sequence regeneration except one study which reported that randomization was done but without giving out how they did it [57], allocation concealment except three studies which implemented random slides for examination $[47,62,63]$, implementation, and blinding. Concerning other items, most studies followed guidelines for reporting preclinical in vitro studies (see supplementary material IV for the detailed assessment).

There are six domains to illustrate the risk of bias in animal studies. The selection domain results showed that not all in vivo studies performed allocation concealment. However, 4 of 6 studies performed randomization and provided similar baseline characteristics of experimental groups $[48,52,67,68]$. The confounding domain was not applicable in the current systematic review as we excluded any studies that have confounding factors during the first phase of selection. The finding of the performance domain revealed that all studies showed identical experimental conditions among study groups. In contrast, only one study reported that independent researchers performed outcome analysis and gave details about the conditions of animals after the experiment [48]. Furthermore, all studies have a low risk of bias in detecting, reporting, and using appropriate statistical analysis domains. One paper did not use statistical analysis [52] (Figures 2 and 3).

3.5. Synthesis of Results. For the proliferation rate, the included studies showed four results: GMSCs had a significantly higher proliferation rate than other MSCs $(n=10)$, GMSCs had an effective proliferation rate somewhat similar to other MSCs $(n=7)$, GMSCs had a lower proliferation rate than other MSCs $(n=0)$, and the proliferation rate was unreported $(n=3)$. Regarding the characterization of interventions, the results of the included studies were identified based on three ways of identifications: morphological, functional, and phenotypical features. Data were presented as follows: definitely sufficient ways used to characterize the intervention ( 3 out 3 ) ( $n=14$ in vitro and 4 in vivo), probably sufficient ways used to characterize the intervention (2 out 3 ) ( $n=3$ in vitro and 2 in vivo), and insufficient ways used to characterize the intervention ( 1 out of 3$)(n=3$ in vitro).

The primary outcome of in vitro culture studies was described as follows: GMSCs had stronger osteogenic potential than other MSCs $(n=2)$, GMSCs had similar osteogenic 


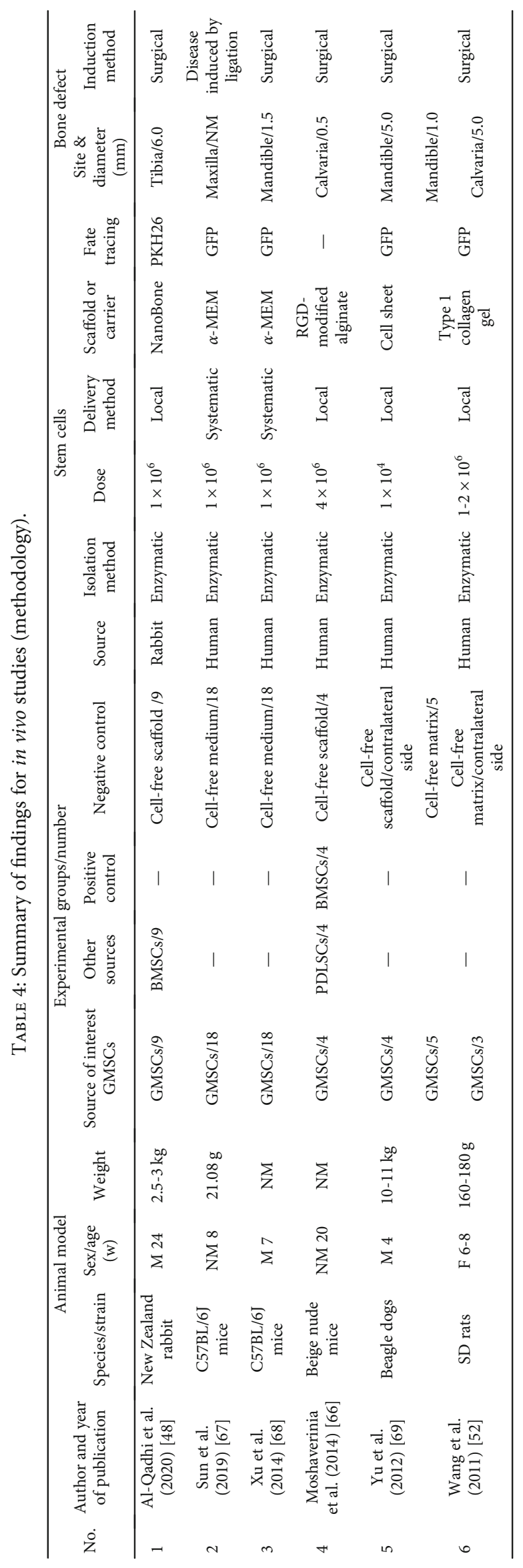




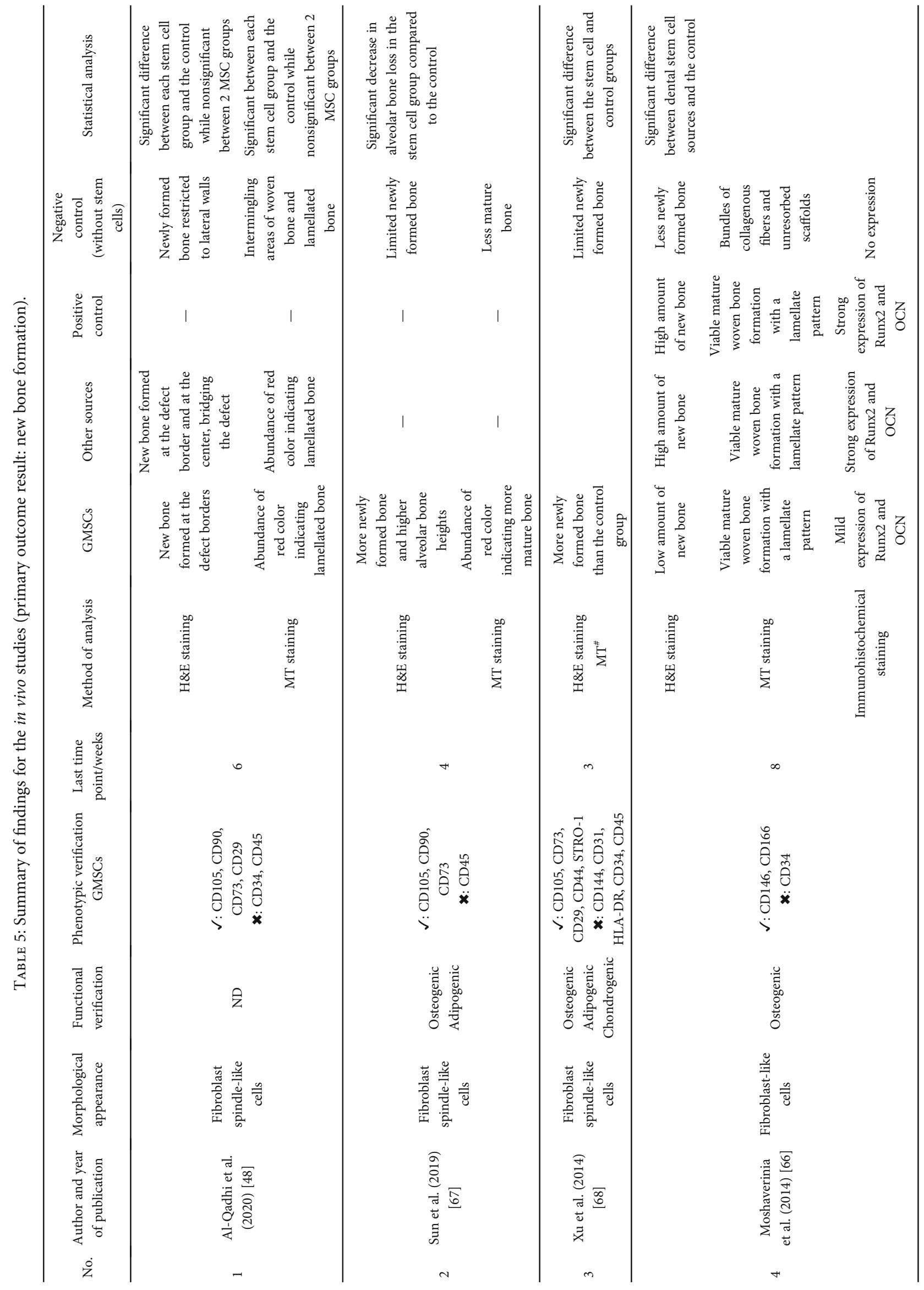




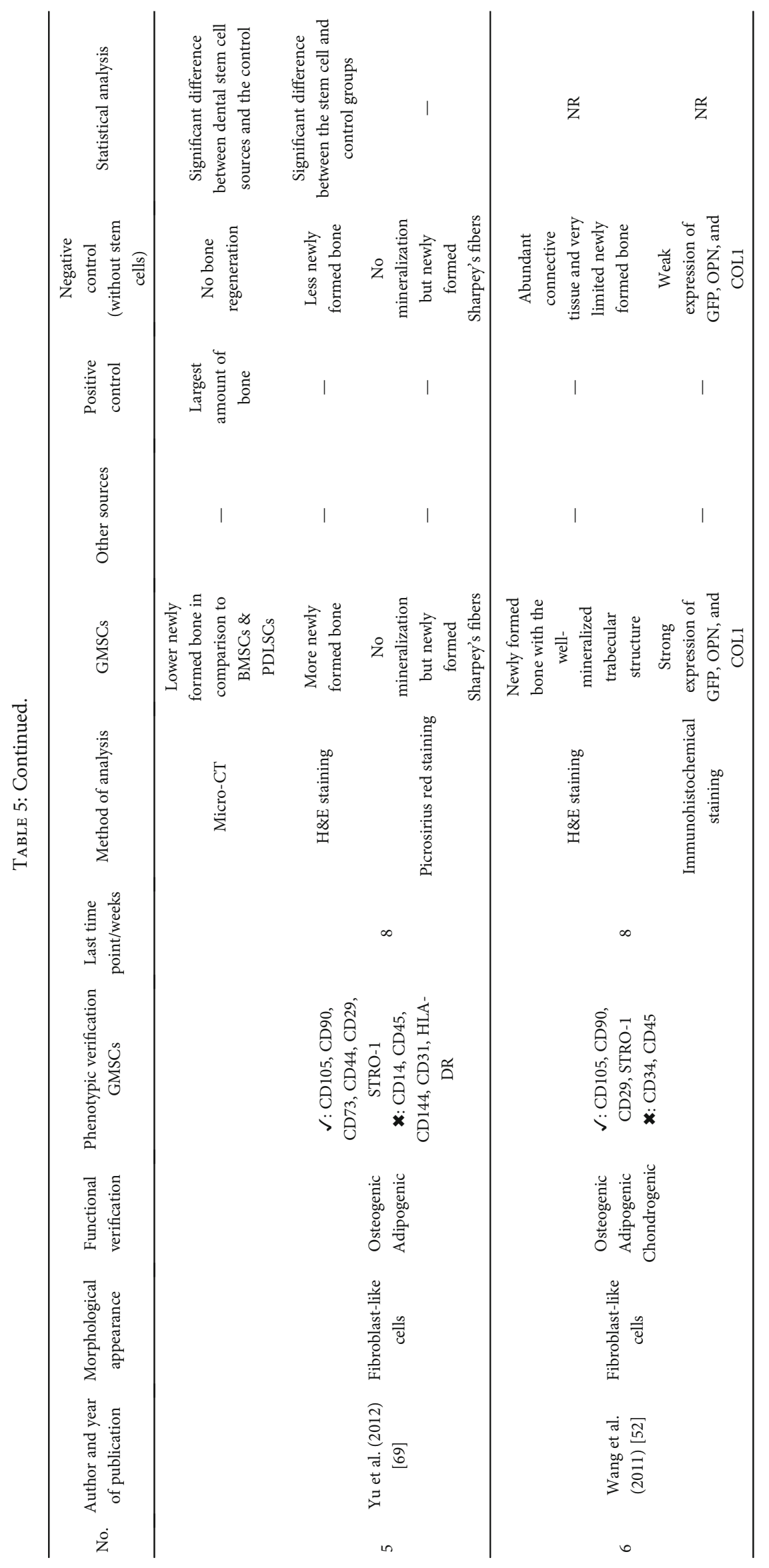




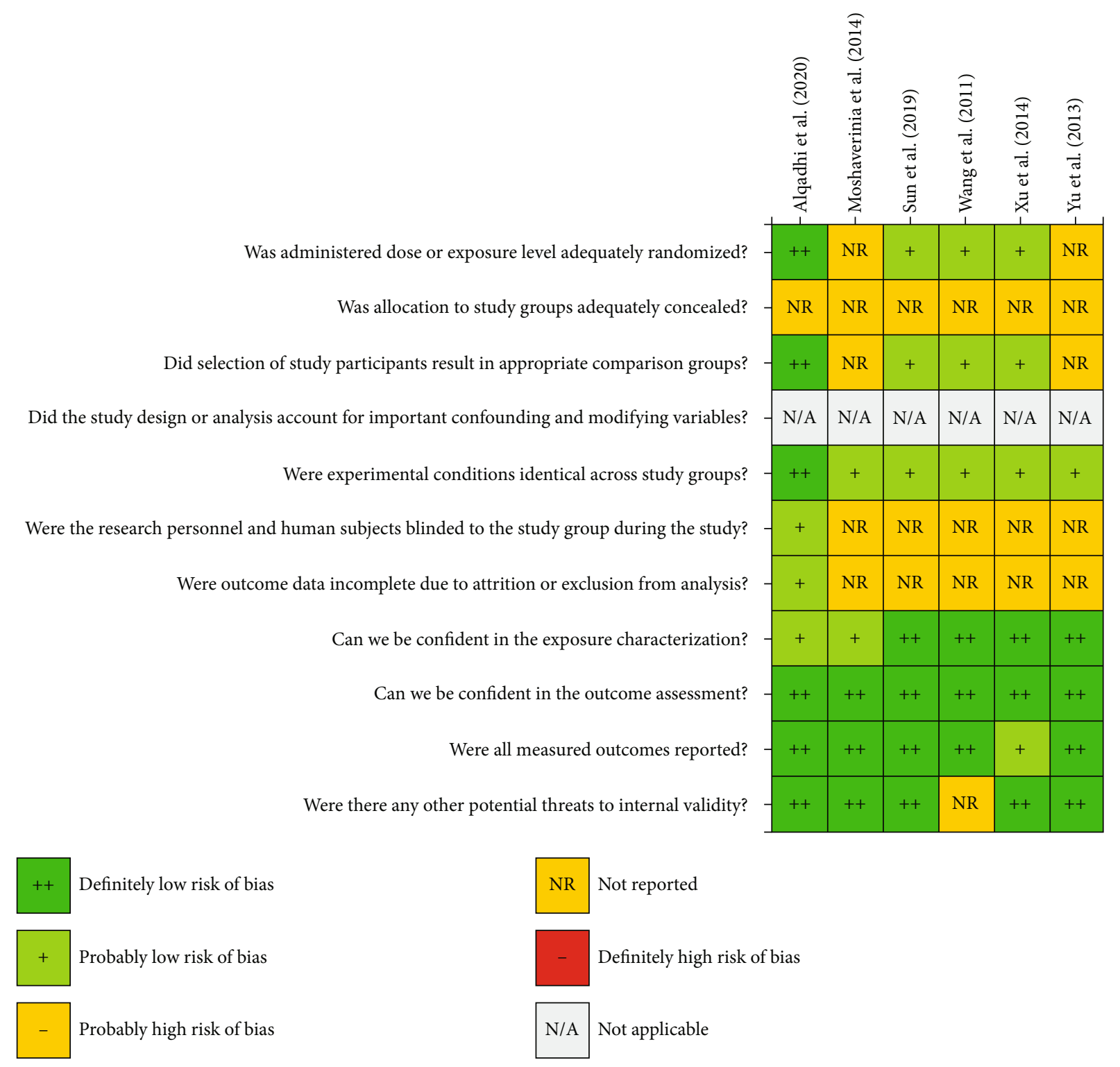

FIGURE 2: An assessment of the risk of bias among animal studies.

potential to other MSCs $(n=8)$, and GMSCs had less osteogenic potential than other MSCs $(n=8)$. Along the same line, the in vivo outcome was presented as follows: more newly formed bone $(n=4)$, comparable newly formed bone $(n=1)$, and less newly formed bone $(n=1)$ (Figure 4$)$.

\section{Discussion}

The main purpose of this systematic review was to summarize the role of gingiva-derived mesenchymal stem cells (GMSCs) and their effects on osteodifferentiation and bone regeneration both in vitro and in vivo. Overall, 26 studies were qualitatively reviewed. Twenty in vitro culture studies and six in vivo studies were mainly carried out to evaluate the proliferation and osteodifferentiation potential of GMSCs in comparison to other sources of MSCs or to nontreated GMSCs. The gingiva is recognized as a biological oral barrier against different insults and shows rapid regeneration with- out scar formation. This ability ascribes to the presence of an abundance of highly proliferative cells. These unique cell populations were firstly identified by Zhang and his colleagues as a cell population with similar stem cell-like properties [28].

Currently, these cells are commonly referred to as GMSCs and considered desirable stem cell sources because of their easy accessibility with limited morbidity. The multipotent differentiation capability of GMSCs has also been compared in vivo and in vitro with other stem cell types such as BMSCs, DPSCs, and PDLSCs. Therefore, the authors of this current review believe that in-depth assessment of the literature on preclinical in vivo and in vitro culture studies of GMSCs in bone tissue engineering and regenerative medicine is of great importance to explore the efficacy of GMSCs as a therapeutic source of MSCs.

The use of MSCs in clinical application generally depends on their biological properties including stemness and 


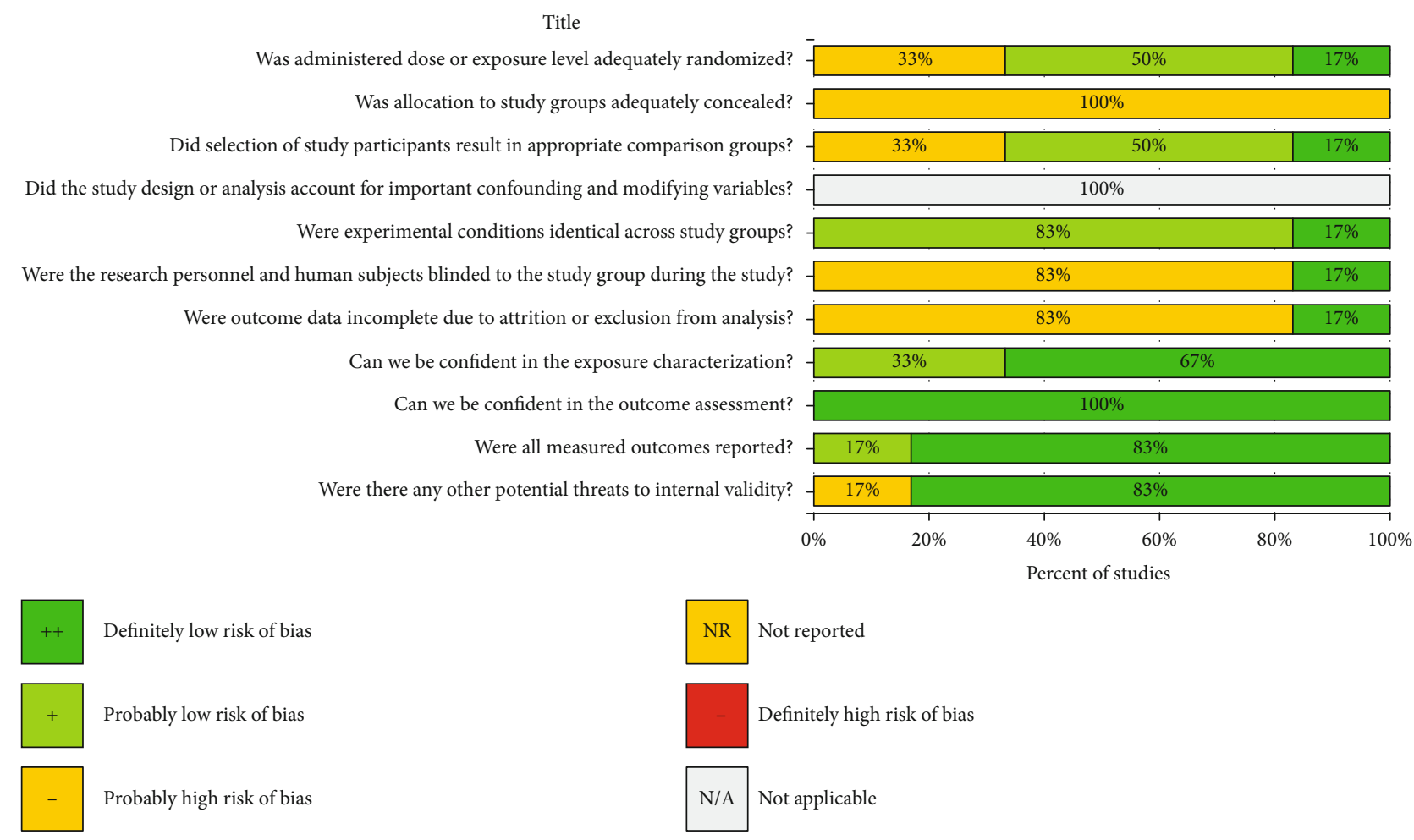

FIGURE 3: A study evaluation bar chart showing the percent of studies with each score, for each metric, in animal studies.

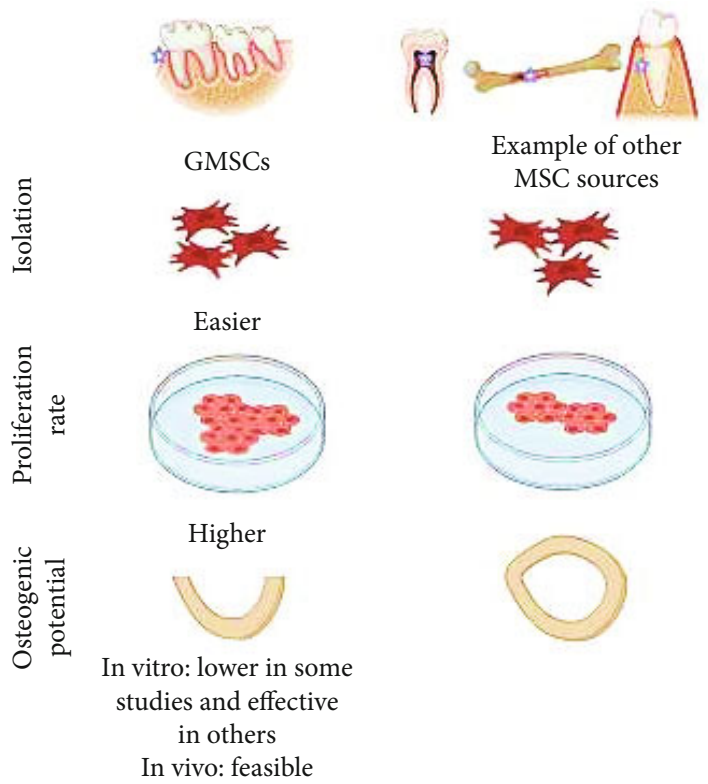

FIGURE 4: Schematic diagram represents the main finding of the current review.

production of healing secretion factors. MSC is commonly isolated as a plastic-adherent cell population via either the enzymatic digestion method or the nonenzymatic digestion method. Researchers believe that cell isolation and expansion protocols influence stem cell quality, including yield, viability, and differentiation potential. Gingival tissues are com- monly obtained after surgical removal of gingival tissue samples and either kept intact to grow out the adherent cells or digested by the use of specific enzymes to obtain single-cell suspensions.

Although no study to compare the two common isolation protocols for GMSCs is available to date, the majority of studies in this review display that GMSCs rapidly proliferate after both isolation methods, irrespective of their species. Likewise, in all studies evaluated in the present review, no study attempts to correlate this superior proliferative ability to any specific isolation method. On average, an increased number of cells with highly proliferative properties from the gingiva appeared after the enzymatic method. Among the studies evaluated in this systematic review (only three studies), a homogeneous population of stem cells was further selected using single-cell cloning [52], fluorescence-activated cell sorting [49], and colony-forming units [51].

Nevertheless, these data might hinder our precise conclusion of the advantages of isolation and purification methods on GMSC quality. In particular, since MSC quality is influenced by several factors during isolation and expansion producers, we recommend further assessment of GMSC isolation and expansion and establish a standard protocol before translating GMSCs to the clinical application. However, a review article by El-Sayed and Dörfer summarized various protocols of GMSC isolation and expansion and addressed some valuable information to achieve MSCs from gingival tissue [27].

To characterize GMSCs and compare their stemness properties to other types of stem cells in vitro, most of the current reviewed studies met the minimal criteria proposed 
by the International Society for Cellular Therapy for MSC characterization [31]. Self-renewal ability as a result of either asymmetrical or symmetrical cell division into different cells with distinct properties is considered the first basic cellular characteristic of stem cells. Almost all reviewed studies showed that GMSCs remain in a quiescent state after isolation and then propagate into a spindle-shaped morphology, similar to BMSCs, DPSCs, DPLSCs, ASCs, and others [46].

The morphological analysis of GMSCs showed no discernible difference between the reviewed studies as GMSCs displayed a stable phenotype in long-term culture and are nontumorigenic [58]. Likewise, GMSCs demonstrated a high ability for colony formation in two-dimensional (2D) culture and high viability rates in RGD-modified alginate hydrogel microspheres similar to BMSCs [54]. Admittedly, MSCs, as primary cells, show some in vitro proliferation potential and differentiation capability to a certain passage before they undergo considerable cellular alterations and senescence $[70,71]$. On the contrary, GMSCs showed some remarkable growth characteristics in comparison to the other stem cell sources. In comparison to BMSCs, Tomar et al. reported that human gingival tissues generated highly proliferative, homogenous fibroblast spindle-like cells with a normal diploid number of chromosomes and normal karyotype even in the late passage [58].

Additionally, human GMSCs display highly growth properties than human BMSCs and PDLSCs, while a short doubling time of GMSCs was observed [62, 66]. GMSCs manifested higher proliferative rates than DPSCs at an early passage with more resistance to induced-oxidative stress and aging in long-term culture [61]. These significant properties are attributed to the continuous activation of the telomerase enzyme in GMSCs even in long-term cultures and to the heterogeneity of other types of stem cells [58].

On the other hand, GMSCs disclosed some unremarkable proliferative rates in comparison to other sources of stem cells, including human BMSCs [62] and pig BMSCs and PSCs [46]. Moreover, the low colony-forming unitfibroblast (CFU-F) ability of GMSCs in comparison to human DSCs was reported [55]. The heterogeneity of the data showed in the current review of the literature can be attributed either to the donor- and tissue-dependent variations or to the cell isolation and expansion procedures. Substantial cell-to-cell variation among MSCs within a single population is thought to play a significant role in the experiments and outcomes [72]. Further studies are needed to investigate the effects of different culture conditions and properties of different GMSC subpopulations.

Nevertheless, the colony-forming assay with increased cell growth demonstrated that GMSCs exhibit a significantly higher proliferation potential than other stem cell sources. This result may be due to the biological function of the gingiva because it shows some exceptional reparative/regenerative potential after wounding. In terms of easy in vitro isolation with a large scale of cell expansion without significant phenotype and genotype alterations, GMSCs can be used as an attractive alternative source to BMSCs in stem cell research studies. Similar to standard characterizations of MSCs from other tissue sources, almost all the reviewed stud- ies showed that GMSCs uniformly expressed certain stem cell markers, including CD90, CD105, CD146, and CD73 (all above 95\%), and did not express hematopoietic stem cell markers CD45 and CD35. Even though the gingiva is densely vascular, these data might confirm the stromal origin of isolated cells without contamination with hematopoietic precursor cells.

However, this might not be necessarily correlated with the ability of MSCs to differentiate into other types of cells, including bone-forming cells. The majority of studies in the current review show the ability of GMSCs for in vitro osteodifferentiation under established osteoblast lineage-specific factors with remarkable changes in protein, gene, and miRNA levels. Unremarkable findings respective to GMSC osteodifferentiation potential in comparison to other stem cell types were also shown. GMSCs displayed less osteogenic potential than other MSC sources, while the high expression level of MSC surface markers was reported [47, 51, 56, 57, 62, 63, 65].

A study by Gao et al. demonstrated that human GMSCs with higher expression of CD90 in comparison to other stem cells of dental origin, DPSCs and PDLSCs, had a moderate osteogenic potential [55]. Two studies showed that GMSCs had better osteogenic capacity than BMSCs and DPSCs $[44,54]$. Successful osteodifferentiation of GMSCs has also been shown in various studies and verified by staining of differentiated cells with Alizarin red or Von Kosssa and/or by expression of bone-related markers including Runx2, ALP, OCN, OPN, OSX, COL1, and COL3 [27]. GMSCs seeded in the 3D culture significantly increased the level of alkaline phosphatase in comparison to those from the 2D culture [73].

The heterogeneity of expression of several MSC surface markers is believed to arise from different subgroups within the MSC population, hence influencing significantly the MSC potency, including their osteodifferentiation potential [74]. In this regard, when correlating the osteogenic properties of GMSCs to the expression of MSC surface markers, in only one in vitro culture study, CD90 expression was evaluated during the osteodifferentiation of both BMSCs and GMSCs. Notably, the expression level of CD90 in BMSCs was gradually lost during their osteodifferentiation, while it stayed very high in GMSCs with a remarkable mineralized nodule formation [44]. CD90 antigen is one of the important markers of MSCs, and it can possibly serve as an index for CFU-F enrichment with strong osteogenic differentiation potential [75].

CD90 (+) subpopulation from ADSCs was characterized with higher osteogenic potential identified via alkaline phosphatase (ALP) and Alizarin red staining [76]. Although previous studies recommended STRO-1 for the enrichment of MSC-like subpopulation from the gingiva and other connective tissues [29], a low percentage of MSCs and reduced level of STRO-1 during culture expansion were reported [77]. Moreover, although such findings highlighted the significant roles of stem cell markers on selecting GMSCs with higher osteodifferentiation potential, further investigations on the influences of site and donor variations, experimental designs, and isolation methods are necessary. 


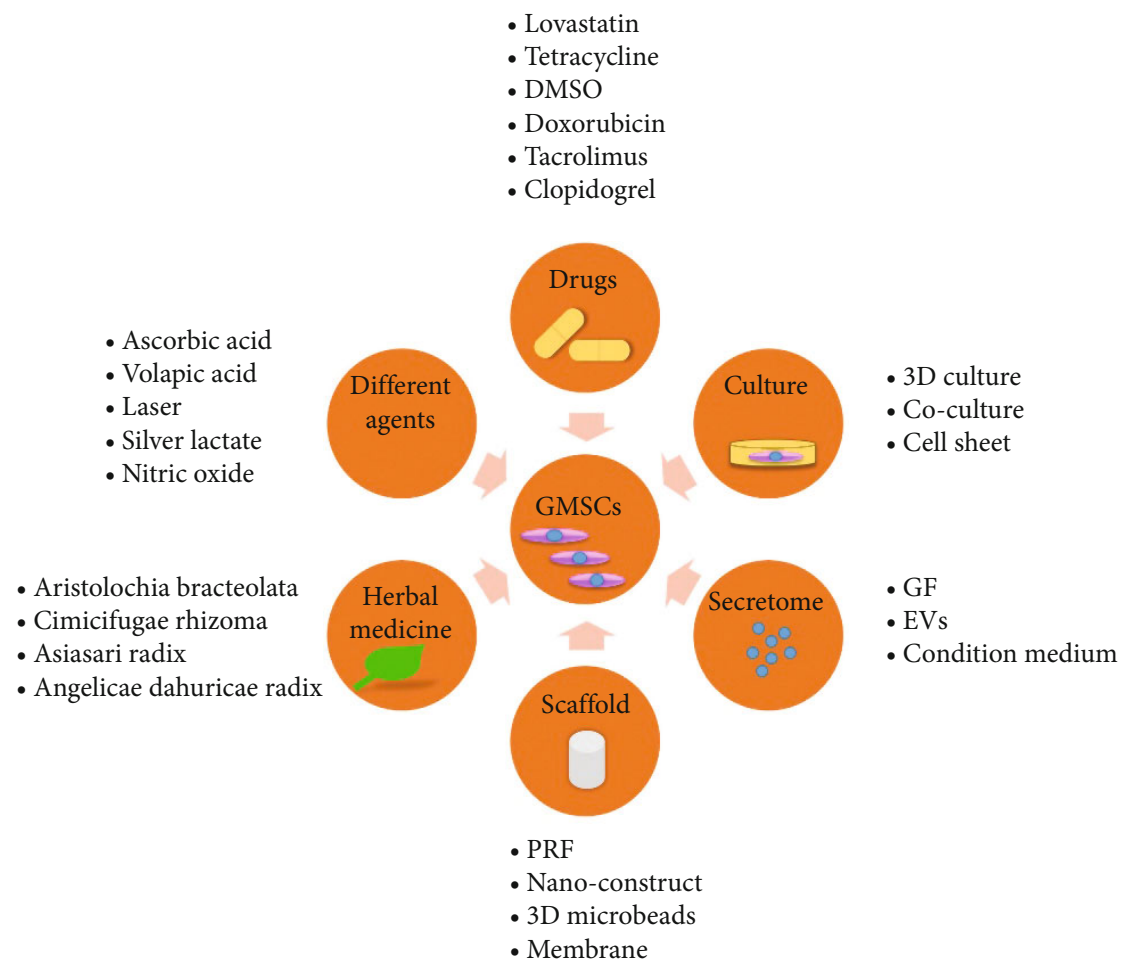

Figure 5: Different factors influence the osteogenic potential of GMSCs.

To research the in vivo bone formation ability of GMSCs in different critical bone defects, in six in vivo studies, GMSCs were either injected systematically or mixed with different carriers before they were locally implanted. Although systematic delivery keeps cells in contact with oxygen and nutrient supply, some research studies approved that it may result in the concentration of MSCs in different sites of the body such as the lung, driven by inflamed organs. The benefit of local transplantation is the close proximity of MSCs to the site of the bone defect. However, the survival of MSCs may be affected due to insufficient oxygen and nutrients at the sites of injection. Therefore, further approaches should be applied to enhance cell engraftment and survival upon therapeutic transplantations [78].

In animal studies included in the current review, almost all GMSCs formed new bone tissues with a lamellate pattern yet in different degrees. Wang et al. demonstrated that GMSCs loaded on collagen scaffolds have more expression of OPN and type 1 collagen when they were implanted in critical-sized mandibular and calvarial defects in rats than the free-cell scaffold group. Moreover, the newly formed bone was detected in both models eight weeks postoperatively [52].

Similarly, GMSCs were implanted systematically via the tail vein into mandibular bone defects in mice; after three weeks of transplantation, new bone formation was significantly higher in the GMSC-loaded group than the unloaded one [68]. In a recent study, Al-Qadhi et al. also reported that GMSCs loaded on NanoBone were able to regenerate bone tissue in critical-sized bone defects in rabbits in a way comparable to BMSCs [48]. Admittedly, a desirable cell carrier should provide the biological template needed for cellular growth, differentiation, and tissue formation [79]. Despite the lower expression of bone-forming markers of GMSCs in comparison to that of BMSCs or PDLSCs, encapsulated GMSCs in RGD-modified alginate microspheres showed an ability to form new bone tissue after 8 weeks in a criticalsized calvarial defect in mice [66]. Ten weeks after implant recovery, GMSC- and BMSC-seeded biografts also showed a higher mineralized tissue with strong OCN expression than the unseeded one [58].

In particular, GMSCs mixed with other cells in one culture or one construct lead to enhanced osteogenic differentiation $[80,81]$. It is therefore important to realize that many factors might influence the regenerative capacity of MSCs, including different culture conditions and cell carriers. Indeed, various articles have demonstrated the positive or negative impact of different factors on the viability and osteogenic potential of GMSCs. These factors include the following: conditioned medium [82], growth factors [83, 84], drugs $[85,86]$, herbal medicine [87], and scaffold type [88] (Figure 5). However, studies using exogenous factors or coculture systems were excluded from this systematic review to precisely conclude the osteodifferentiation potential of GMSCs without any synergistic or confounding factors.

\section{Limitation in the Current Reviewed Studies}

Even though in vitro culture preclinical research studies are considered a basic step for future research or for any novel therapeutic approach, it is worth mentioning that the methodological quality analysis of all evaluated in vitro culture 
studies shows some bias, such as lack of repetition of the experiments and lack of randomization, blinding, and sample size calculation. In spite of these factors that can influence the scientific validity of experimental results as all phases of the research process are interlinked, some researchers thought that those quality analyses could not be applied to in vitro culture studies [89]. For instance, the insufficient sample size might give incorrect results, which then may affect the outcomes of preclinical studies and in turn future clinical trials. We, therefore, found it is reasonable to apply the modified CONSORT guidelines [41] to in vitro culture studies to emphasize the importance of avoiding bias in future MSC research studies.

Importantly, no clinical trials of bone tissue engineering by GMSCs were found. The authors believe that the reasons might ascribe either to an early judgment of the outcomes from preclinical studies or to insufficient data collected. To confirm whether the outcomes of in vivo studies are not influenced by intended or unintended bias, all animal studies should have a clear study design. These parameters should include reporting of randomization, baseline characteristics (age, sex, and weight), animal housing conditions, blinding, animals enrolled and attrition, defect characteristics, followup, disclosing any adverse effects during and after the intervention, sample size, methods of size calculation, and reporting statistical analysis. This information will significantly improve the internal and external validity of any study and will also help researchers to publish a high level of scientific evidence similar to human RCTs.

With respect to the in vivo studies, most studies revealed a probably low risk of bias in sequence, detection, reporting, and one metric of performance (identical across group study) domains, while another metric of performance (blinding) and attrition domains were not reported.

\section{Conclusion}

Although GMSCs show similar or lower osteodifferentiation potential in vitro culture studies compared to other MSC sources, regenerating bony defects in vivo was significantly feasible with GMSCs. The easy accessibility and highly proliferative ability of GMSCs with a short doubling time could make them an attractive alternative source in the field of bone tissue engineering. However, the limited in vitro degree of osteodifferentiation potential of GMSCs remains a disadvantageous outcome. Therefore, further optimization of the in vitro culture conditions is needed.

In the same way, due to the insufficient number of in vivo studies that highlight the role of GMSCs in bone regeneration and based on the quality of the literature, using appropriate animal models with critical bone defects rather than ectopic models is highly important before going into a clinical application. Despite such findings for the benefit of using GMSCs in bone regeneration, well-designed preclinical studies that follow rigorous guidelines and manage a range of conditions such as experimental models, differentiating factors, culture media, and biological activity, cost-effectiveness, and safety of GMSCs are required.

\section{Conflicts of Interest}

The authors declare that there is no conflict of interest regarding the publication of this paper.

\section{Supplementary Materials}

The current research has the following supplementary materials:

Supplementary 1. Supplementary material I. PRISMA checklist.

Supplementary 2. Supplementary material II. Detailed search strategy.

Supplementary 3. Supplementary material III. List of excluded studies.

Supplementary 4. Supplementary material IV. Detailed in vitro assessment.

\section{References}

[1] G. Fernandez de Grado, L. Keller, Y. Idoux-Gillet et al., "Bone substitutes: a review of their characteristics, clinical use, and perspectives for large bone defects management," Journal of tissue engineering, vol. 9, 2018.

[2] A. Oryan, S. Alidadi, A. Moshiri, and N. Maffulli, "Bone regenerative medicine: classic options, novel strategies, and future directions," Journal of Orthopaedic Surgery and Research, vol. 9, no. 1, pp. 18-27, 2014.

[3] W. Wang and K. W. K. Yeung, "Bone grafts and biomaterials substitutes for bone defect repair: a review," Bioactive Materials, vol. 2, no. 4, pp. 224-247, 2017.

[4] S. Govender, C. Csimma, H. K. Genant et al., "Recombinant human bone morphogenetic protein-2 for treatment of open tibial fractures: a prospective, controlled, randomized study of four hundred and fifty patients," The Journal of Bone and Joint Surgery, vol. 84, no. 12, pp. 2123-2134, 2002.

[5] T. J. Webster, C. Ergun, R. H. Doremus, R. W. Siegel, and R. Bizios, "Specific proteins mediate enhanced osteoblast adhesion on nanophase ceramics," Journal of Biomedical Materials Research, vol. 51, no. 3, pp. 475-483, 2000.

[6] G. Mendonça, D. B. S. Mendonça, F. J. L. Aragão, and L. F. Cooper, "Advancing dental implant surface technology - from micron- to nanotopography," Biomaterials, vol. 29, no. 28, pp. 3822-3835, 2008.

[7] W. Paiva, D. Sola, T. Paiva, and M. Andre, "Potential toxicity of dental nanomaterials to the central nervous system," International Journal of Nanomedicine, vol. 10, pp. 5593-5594, 2015.

[8] A. Ballini, S. Cantore, S. Scacco, D. Coletti, and M. Tatullo, "Mesenchymal stem cells as promoters, enhancers, and playmakers of the translational regenerative medicine 2018," Stem Cells International, vol. 2018, Article ID 6927401, 2 pages, 2018.

[9] Q. Zhao, H. Ren, and Z. Han, "Mesenchymal stem cells: immunomodulatory capability and clinical potential in immune diseases," Journal of Cellular Immunotherapy, vol. 2, no. 1, pp. 3-20, 2016.

[10] Y. Shi, Y. Wang, Q. Li et al., "Immunoregulatory mechanisms of mesenchymal stem and stromal cells in inflammatory 
diseases," Nature Reviews. Nephrology, vol. 14, no. 8, pp. 493507, 2018.

[11] T. L. Arinzeh, T. Tran, J. Mcalary, and G. Daculsi, “A comparative study of biphasic calcium phosphate ceramics for human mesenchymal stem-cell-induced bone formation," Biomaterials, vol. 26, no. 17, pp. 3631-3638, 2005.

[12] H. Kim, H. Suh, S. A. Jo et al., "In vivo bone formation by human marrow stromal cells in biodegradable scaffolds that release dexamethasone and ascorbate-2-phosphate," Biochemical and Biophysical Research Communications, vol. 332, no. 4, pp. 1053-1060, 2005.

[13] K. A. Blackwood, N. Bock, T. R. Dargaville, and M. Ann Woodruff, "Scaffolds for growth factor delivery as applied to bone tissue engineering," International Journal of Polymer Science, vol. 2012, Article ID 174942, 25 pages, 2012.

[14] M. Tatullo, G. Spagnuolo, B. Codispoti et al., "PLA-based mineral-doped scaffolds seeded with human periapical cyst-derived MSCs: a promising tool for regenerative healing in dentistry," Materials (Basel), vol. 12, no. 4, pp. 597617, 2019.

[15] G. Turnbull, J. Clarke, F. Picard et al., "3D bioactive composite scaffolds for bone tissue engineering," Bioactive Materials, vol. 3, no. 3, pp. 278-314, 2018.

[16] M. Marrelli, B. Codispoti, R. M. Shelton et al., "Dental pulp stem cell mechanoresponsiveness: effects of mechanical stimuli on dental pulp stem cell behavior," Frontiers in physiology, vol. 9, 2018.

[17] L. Polo-Corrales, M. Latorre-Esteves, and J. E. Ramirez-Vick, "Scaffold design for bone regeneration," Journal of Nanoscience and Nanotechnology, vol. 14, no. 1, pp. 15-56, 2014.

[18] P. Giannoni, M. Alini, S. G. Pearce et al., "Regeneration of large bone defects in sheep using bone marrow stromal cells," Journal of Tissue Engineering and Regenerative Medicine, vol. 2, no. 5, pp. 253-262, 2008.

[19] D. Li, M. Li, P. Liu, Y. Zhang, J. Lu, and J. Li, “Tissue-engineered bone constructed in a bioreactor for repairing criticalsized bone defects in sheep," International Orthopaedics, vol. 38, no. 11, pp. 2399-2406, 2014.

[20] L. Lu, Y. Liu, X. Zhang, and J. Lin, "The therapeutic role of bone marrow stem cell local injection in rat experimental periodontitis," Journal of Oral Rehabilitation, vol. 47, no. S1, pp. 73-82, 2019.

[21] F. Chamieh, A. M. Collignon, B. R. Coyac et al., “Accelerated craniofacial bone regeneration through dense collagen gel scaffolds seeded with dental pulp stem cells," Scientific reports, vol. 6, no. 1, pp. 1-11, 2016.

[22] Y. Yamada, S. Nakamura-Yamada, R. Konoki, and S. Baba, "Promising advances in clinical trials of dental tissue-derived cell-based regenerative medicine," Stem Cell Research and Therapy, vol. 11, no. 1, article 1683, p. 175, 2020.

[23] H.-T. Liao and C. T. Chen, "Osteogenic potential: comparison between bone marrow and adipose-derived mesenchymal stem cells," World journal of stem cells, vol. 6, no. 3, pp. 288295, 2014.

[24] G. Spagnuolo, B. Codispoti, M. Marrelli, C. Rengo, S. Rengo, and M. Tatullo, "Commitment of oral-derived stem cells in dental and maxillofacial applications," Dental Journal, vol. 6, no. 4, pp. 72-78, 2018.

[25] L. Tomasello, A. Coppola, M. Pitrone et al., "Mesenchymal stem cells derived from inflamed dental pulpal and gingival tis- sue: a potential application for bone formation," Stem cell research \& therapy, vol. 8, no. 1, p. 179, 2017.

[26] H. Egusa, K. Okita, H. Kayashima et al., "Gingival fibroblasts as a promising source of induced pluripotent stem cells," PLoS One, vol. 5, no. 9, article e12743, 2010.

[27] K. M. Fawzy El-Sayed and C. E. Dörfer, "Gingival mesenchymal stem/progenitor cells: a unique tissue engineering gem," Stem Cells International, vol. 2016, Article ID 7154327, 16 pages, 2016.

[28] Q. Zhang, S. Shi, Y. Liu et al., "Mesenchymal stem cells derived from human gingiva are capable of immunomodulatory functions and ameliorate inflammation-related tissue destruction in experimental colitis," Journal of Immunology, vol. 183, no. 12, pp. 7787-7798, 2009.

[29] K. M. F. el-Sayed, S. Paris, C. Graetz et al., "Isolation and characterisation of human gingival margin-derived STRO$1 / \mathrm{MACS}^{+}$and $\mathrm{MACS}^{-}$cell populations," International Journal of Oral Science, vol. 7, no. 2, pp. 80-88, 2015.

[30] X. Xu, C. Chen, K. Akiyama et al., "Gingivae contain neuralcrest- and mesoderm-derived mesenchymal stem cells," Journal of Dental Research, vol. 92, no. 9, pp. 825-832, 2013.

[31] M. Dominici, K. le Blanc, I. Mueller et al., "Minimal criteria for defining multipotent mesenchymal stromal cells. The International Society for Cellular Therapy position statement," Cytotherapy, vol. 8, no. 4, pp. 315-317, 2006.

[32] S. Ansari, I. M. Diniz, C. Chen et al., "Human periodontal ligament- and gingiva-derived mesenchymal stem cells promote nerve regeneration when encapsulated in alginate/hyaluronic acid 3D scaffold," Advanced healthcare materials, vol. 6, no. 24, article 1700670, 2017.

[33] Q. Mao, P. D. Nguyen, R. M. Shanti et al., "Gingivaderived mesenchymal stem cell-extracellular vesicles activate Schwann cell repair phenotype and promote nerve regeneration," Tissue Engineering Part A, vol. 25, no. 1112, pp. 887-900, 2019.

[34] P. E. Bunney, A. N. Zink, A. A. Holm, C. J. Billington, and C. M. Kotz, "Orexin activation counteracts decreases in nonexercise activity thermogenesis (NEAT) caused by high-fat diet," Annals of Biomedical Engineering, vol. 176, no. 6, pp. 139-148, 2016.

[35] A. Moshaverinia, X. Xu, C. Chen et al., "Application of stem cells derived from the periodontal ligament or gingival tissue sources for tendon tissue regeneration," Biomaterials, vol. 35, no. 9, pp. 2642-2650, 2014.

[36] Q. Shi, Z. Qian, D. Liu et al., "GMSC-derived exosomes combined with a chitosan/silk hydrogel sponge accelerates wound healing in a diabetic rat skin defect model," Frontiers in physiology, vol. 8, 2017.

[37] F. C. Ferré, H. Larjava, L. S. Loison-Robert et al., "Formation of cartilage and synovial tissue by human gingival stem cells," Stem Cells and Development, vol. 23, no. 23, pp. 2895-2907, 2014.

[38] E. S. Sena, G. L. Currie, S. K. McCann, M. R. Macleod, and D. W. Howells, "Systematic reviews and meta-analysis of preclinical studies: why perform them and how to appraise them critically," Journal of Cerebral Blood Flow and Metabolism, vol. 34, no. 5, pp. 737-742, 2014.

[39] D. Moher, A. Liberati, J. Tetzlaff, D. G. Altman, and The PRISMA Group, "Preferred reporting items for systematic reviews and meta-analyses: the PRISMA statement," PLOS Medicine, vol. 6, no. 7, article e1000097, 2009. 
[40] M. Leenaars, C. R. Hooijmans, N. van Veggel et al., “A step-bystep guide to systematically identify all relevant animal studies," Laboratory Animals, vol. 46, no. 1, pp. 24-31, 2012.

[41] C. M. Faggion, "Guidelines for reporting pre-clinical in vitro studies on dental materials," The Journal of Evidence-Based Dental Practice, vol. 12, no. 4, pp. 182-189, 2012.

[42] C. R. Hooijmans, M. M. Rovers, R. B. M. De Vries, M. Leenaars, M. Ritskes-hoitinga, and M. W. Langendam, "SYRCLE's risk of bias tool for animal studies," BMC Medical Research Methodology, vol. 14, no. 1, article 1067, pp. 1-9, 2014.

[43] A. J. Shapiro, K. Z. Guyton, R. M. Lunn et al., "Software tools to facilitate systematic review used for cancer hazard identification," Environmental health perspectives, vol. 126, no. 10, article 104501, 2018.

[44] Q. Sun, H. Nakata, M. Yamamoto, S. Kasugai, and S. Kuroda, "Comparison of gingiva-derived and bone marrow mesenchymal stem cells for osteogenesis," Journal of Cellular and Molecular Medicine, vol. 23, no. 11, pp. 7592-7601, 2019.

[45] A. Moshaverinia, C. Chen, K. Akiyama et al., "Alginate hydrogel as a promising scaffold for dental-derived stem cells: an in vitro study," Journal of Materials Science. Materials in Medicine, vol. 23, no. 12, pp. 3041-3051, 2012.

[46] N. Kaibuchi, T. Iwata, S. Onizuka et al., "Cytological character of mini pig mesenchymal stromal cells from various tissues and the attempt of cell sheet formation," Regenerative therapy, vol. 6, pp. 83-89, 2017.

[47] N. Mensing, H. Gasse, N. Hambruch, J. D. Haeger, C. Pfarrer, and C. Staszyk, "Isolation and characterization of multipotent mesenchymal stromal cells from the gingiva and the periodontal ligament of the horse," BMC Veterinary Research, vol. 7, no. 1, pp. 42-113, 2011.

[48] G. Al-Qadhi, M. Soliman, I. Abou-Shady, and L. Rashed, "Gingival mesenchymal stem cells as an alternative source to bone marrow mesenchymal stem cells in regeneration of bone defects: in vivo study," Tissue Cell, vol. 63, p. 101325, 2020.

[49] I. Gay, A. Cavender, D. Peto et al., "Differentiation of human dental stem cells reveals a role for microRNA-218," Journal of Periodontal Research, vol. 49, no. 1, pp. 110-120, 2014.

[50] D. Ponnaiyan, K. M. Bhat, and G. S. Bhat, "Comparison of immuno-phenotypes of stem cells from human dental pulp and periodontal ligament," International Journal of Immunopathology and Pharmacology, vol. 25, no. 1, pp. 127134, 2012.

[51] B. P. J. Fournier, F. C. Ferre, L. Couty et al., "Multipotent progenitor cells in gingival connective tissue," Tissue Engineering Part A, vol. 16, no. 9, pp. 2891-2899, 2010.

[52] F. Wang, M. Yu, X. Yan et al., "Gingiva-derived mesenchymal stem cell-mediated therapeutic approach for bone tissue regeneration," Stem Cells and Development, vol. 20, no. 12, pp. 2093-2102, 2011.

[53] H. Ghaderi, M. Razmkhah, F. Kiany, N. Chenari, M. R. Haghshenas, and A. Ghaderi, "Comparison of osteogenic and chondrogenic differentiation ability of buccal fat pad derived mesenchymal stem cells and gingival derived cells," Journal of Dentistry, vol. 19, no. 2, pp. 124-131, 2018.

[54] S. Ansari, P. Sarrion, M. M. Hasani-Sadrabadi, T. Aghaloo, B. $\mathrm{M}$. $\mathrm{Wu}$, and A. Moshaverinia, "Regulation of the fate of dental-derived mesenchymal stem cells using engineered alginate-GelMA hydrogels," Journal of Biomedical Materials Research Part A, vol. 105, no. 11, pp. 2957-2967, 2017.
[55] Y. Gao, G. Zhao, D. Li, X. Chen, J. Pang, and J. Ke, "Isolation and multiple differentiation potential assessment of human gingival mesenchymal stem cells," International Journal of Molecular Sciences, vol. 15, no. 11, pp. 20982-20996, 2014.

[56] I. Angelopoulos, C. Brizuela, and M. Khoury, "Gingival mesenchymal stem cells outperform haploidentical dental pulpderived mesenchymal stem cells in proliferation rate, migration ability, and angiogenic potential," Cell Transplantation, vol. 27, no. 6, pp. 967-978, 2018.

[57] Y. Zhang, Y. Xing, L. Jia et al., "An in vitro comparative study of multisource derived human mesenchymal stem cells for bone tissue engineering," Stem Cells and Development, vol. 27, no. 23, pp. 1634-1645, 2018.

[58] G. B. Tomar, R. K. Srivastava, N. Gupta et al., "Human gingiva-derived mesenchymal stem cells are superior to bone marrow-derived mesenchymal stem cells for cell therapy in regenerative medicine," Biochemical and Biophysical Research Communications, vol. 393, no. 3, pp. 377-383, 2010.

[59] Z. Abedian, A. A. Moghadamnia, E. Zabihi et al., "A comparative study on immunophenotypic characterization and osteogenic differentiation of human mesenchymal stromal cells derived from periodontal ligament and gingiva," Journal of periodontology, vol. 91, no. 9, pp. 1194-1202, 2020.

[60] H. Yang, L. N. Gao, Y. An et al., "Comparison of mesenchymal stem cells derived from gingival tissue and periodontal ligament in different incubation conditions," Biomaterials, vol. 34, no. 29, pp. 7033-7047, 2013.

[61] Y. Xing, Y. Zhang, X. Wu, B. Zhao, Y. Ji, and X. Xu, “A comprehensive study on donor-matched comparisons of three types of mesenchymal stem cells-containing cells from human dental tissue," Journal of Periodontal Research, vol. 54, no. 3, pp. 286-299, 2019.

[62] I. M. Aboushady, Z. A. Salem, D. Sabry, and A. Mohamed, "Comparative study of the osteogenic potential of mesenchymal stem cells derived from different sources," Journal of Clinical and Experimental Dentistry, vol. 10, no. 1, pp. e7-e13, 2018.

[63] V. L. Zorin, V. S. Komlev, A. I. Zorina et al., "Octacalcium phosphate ceramics combined with gingiva-derived stromal cells for engineered functional bone grafts," Biomedical materials, vol. 9, no. 5, article 055005, 2014.

[64] R. Monterubbianesi, M. Bencun, P. Pagella, A. Woloszyk, G. Orsini, and T. A. Mitsiadis, "A comparative_in vitro_study of the osteogenic and adipogenic potential of human dental pulp stem cells, gingival fibroblasts and foreskin fibroblasts," Journal of Stem Cells, vol. 9, no. 1, article 37981, pp. 17611830, 2019.

[65] A. Moshaverinia, K. Akiyama, X. Xu et al., "Encapsulated dental-derived mesenchymal stem cells in an injectable and biodegradable scaffold for applications in bone tissue engineering," Journal of Biomedical Materials Research Part A, vol. 101, no. 11, pp. 3285-3294, 2013.

[66] A. Moshaverinia, C. Chen, X. Xu et al., "Bone regeneration potential of stem cells derived from periodontal ligament or gingival tissue sources encapsulated in RGD-modified alginate scaffold," Tissue Engineering Part A, vol. 20, no. 3-4, pp. 131106060201007-131106060201621, 2014.

[67] W. Sun, Z. Wang, Q. Xu et al., "The treatment of systematically transplanted gingival mesenchymal stem cells in periodontitis in mice," Experimental and therapeutic medicine, vol. 17, no. 3, pp. 2199-2205, 2019. 
[68] Q. C. Xu, Z. G. Wang, Q. X. Ji et al., “Systemically transplanted human gingiva-derived mesenchymal stem cells contributing to bone tissue regeneration," International Journal of Clinical and Experimental Pathology, vol. 7, no. 8, pp. 4922-4929, 2014.

[69] X. Yu, S. Ge, S. Chen et al., "Human gingiva-derived mesenchymal stromal cells contribute to periodontal regeneration in beagle dogs," Cells, Tissues, Organs, vol. 198, no. 6, pp. 428-437, 2013.

[70] Z. Li, C. Liu, Z. Xie et al., "Epigenetic dysregulation in mesenchymal stem cell aging and spontaneous differentiation," PLoS One, vol. 6, no. 6, article e20526, 2011.

[71] Y. Wu and R. C. H. Zhao, "The role of chemokines in mesenchymal stem cell homing to myocardium," Stem cell reviews and reports, vol. 8, no. 1, article 9293, pp. 243-250, 2012.

[72] C. McLeod and R. L. Mauck, "On the origin and impact of mesenchymal stem cell heterogeneity: new insights and emerging tools for single cell analysis," European cells \& materials, vol. 34, pp. 217-231, 2017.

[73] S. I. Lee, Y. Ko, and J. B. Park, "Evaluation of the osteogenic differentiation of gingiva-derived stem cells grown on culture plates or in stem cell spheroids: comparison of two- and three-dimensional cultures," Experimental and Therapeutic Medicine, vol. 14, no. 3, pp. 2434-2438, 2017.

[74] L. Du, P. Yang, and S. Ge, "Isolation and characterization of human gingiva-derived mesenchymal stem cells using limiting dilution method," Journal of dental sciences, vol. 11, no. 3, pp. 304-314, 2016.

[75] D. A. Moraes, L. F. Pavon, P. Q. Alvim et al., “A reduction in CD90 (THY-1) expression results in increased differentiation of mesenchymal stromal cells," Stem Cell Research \& Therapy, vol. 7, no. 1, pp. 97-114, 2016.

[76] M. T. Chung, C. Liu, J. S. Hyun et al., "CD90 (Thy-1)-positive selection enhances osteogenic capacity of human adiposederived stromal cells," Tissue Engineering Part A, vol. 19, no. 7-8, pp. 989-997, 2013.

[77] F. J. Lv, R. S. Tuan, K. M. C. Cheung, and V. Y. L. Leung, "Concise review: the surface markers and identity of human mesenchymal stem cells," Stem Cells, vol. 32, no. 6, pp. 14081419, 2014.

[78] Y. Safarova, B. Umbayev, G. Hortelano, and S. Askarova, "Mesenchymal stem cells modifications for enhanced bone targeting and bone regeneration," Regenerative Medicine, vol. 15, no. 4, pp. 1579-1594, 2020.

[79] M. Horst, S. Madduri, R. Gobet, T. Sulser, H. Hall, and D. Eberli, "Scaffold characteristics for functional hollow organ regeneration," Materials (Basel), vol. 3, no. 1, pp. 241-263, 2010.

[80] S. Treves-Manusevitz, H. Rachima, G. Montoya et al., "Stem cells of the lamina propria of human oral mucosa and gingiva develop into mineralized tissues in vivo," Journal of Clinical Periodontology, vol. 40, no. 1, pp. 73-81, 2013.

[81] S. Proksch, K. Kirsch, K. Vach, E. Hellwig, and P. Tomakidi, "Comparative differentiation analysis of distinct oral tissuederived cells in response to osteogenic stimulation," Clinical Oral Investigations, vol. 23, no. 3, pp. 1077-1089, 2019.

[82] F. Diomede, D. Scionti, I. Merciaro et al., "Biotherapeutic effect of gingival stem cells conditioned medium in bone tissue restoration," International journal of molecular sciences, vol. 19, no. 2, p. 329, 2018.

[83] X. Chen, Y. Hou, P. Song et al., "Modulation of proliferation and differentiation of gingiva-derived mesenchymal stem cells by concentrated growth factors: potential implications in tissue engineering for dental regeneration and repair," International Journal of Molecular Medicine, vol. 44, no. 1, pp. 3746, 2019.

[84] H. Lee, S. Min, Y. Song, Y. Park, and J. Park, "Bone morphogenetic protein-7 upregulates genes associated with osteoblast differentiation, including collagen I, Sp7 and IBSP in gingivaderived stem cells," Experimental and therapeutic medicine, vol. 18, no. 4, pp. 2867-2876, 2019.

[85] S. H. Jin, H. Y. Kweon, J. B. Park, and C. H. Kim, "The effects of tetracycline-loaded silk fibroin membrane on proliferation and osteogenic potential of mesenchymal stem cells," The Journal of Surgical Research, vol. 192, no. 2, pp. E1-E9, 2014.

[86] D. H. Ha, C. S. Yong, J. O. Kim, J. H. Jeong, and J. B. Park, "Effects of tacrolimus on morphology, proliferation and differentiation of mesenchymal stem cells derived from gingiva tissue," Molecular Medicine Reports, vol. 14, no. 1, pp. 69-76, 2016.

[87] S. H. Jeong, J. E. Lee, S. H. Jin, Y. Ko, and J. B. Park, "Effect of Asiasari radix on osteoblastic differentiation of stem cells derived from gingiva," Journal of Traditional Chinese Medicine, vol. 36, no. 6, pp. 756-759, 2016.

[88] A. P. Nugraha, I. B. Narmada, D. S. Ernawati et al., "Osteogenic potential of gingival stromal progenitor cells cultured in platelet rich fibrin is predicted by core-binding factor subunit- $\alpha 1 /$ Sox 9 expression ratio (in vitro)," F1000Research, vol. 7, article 1134, p. 1134, 2018.

[89] C. Hirsch and S. Schildknecht, "In vitro research reproducibility: keeping up high standards," Frontiers in pharmacology, vol. 10, pp. 1-9, 2019. 